\title{
Grundsätzlich bereit, aber doch nicht dabei - Eine Analyse der Mitarbeitsbereitschaft in Parteien anhand des Civic Voluntarism Models
}

\author{
Isabelle Borucki $\cdot$ Lena Masch $\cdot$ Simon Jakobs
}

Angenommen: 4. Februar 2021 / Online publiziert: 24. Februar 2021

(C) Der/die Autor(en) 2021

Zusammenfassung Wer sind die Menschen, die sich eine Parteimitgliedschaft vorstellen können, aber nicht beitreten? Mit den Datensätzen der Allgemeinen Bevölkerungsumfrage der Sozialwissenschaften (ALLBUS) der Jahre 2008 und 2018 liegen nunmehr größere, öffentliche und vor allem repräsentative Studien vor, die den Willen zu einer Parteiaktivität ermitteln. Mithilfe dieser Daten wird analysiert, inwieweit sozio-demografische Ressourcen, Motivation, Selbstwirksamkeit und die Einbindung in soziale Netzwerke mit dem Willen, aktiv in Parteien mitzuarbeiten, zusammenhängen. Logistische Regressionsmodelle zeigen nur einen geringen Einfluss soziodemographischer Faktoren. Allerdings sind politische Einstellungen wie beispielsweise das Vertrauen in Parteien relevant. Dies gilt vor allem für die ostdeutschen Bundesländer. Die Ergebnisse deuten darauf hin, dass das Civic Voluntarism Model neben einer Vielzahl von Beteiligungsformen auch - zumindest teilweise dazu geeignet ist, eine beabsichtigte Parteiaktivität zu erklären.

\footnotetext{
Dr. I. Borucki $(\bowtie)$

Institut für Politikwissenschaft, Universität Duisburg-Essen, Lotharstr. 53, 47057 Duisburg,

Deutschland

E-Mail: isabelle.borucki@uni-due.de

Dr. L. Masch

Institut für Sozialwissenschaften, Heinrich-Heine-Universität Düsseldorf, Universitätsstr. 1, 40225 Düsseldorf, Deutschland

E-Mail: lena.masch@uni-duesseldorf.de

Dr. S. Jakobs

Leitung der Akademie, interPartner GmbH, Kennedyplatz 6, 45127 Essen, Deutschland

E-Mail: Simon.jakobs@t-online.de
} 


\title{
Ready, but not there-An analysis of willingness to cooperate in political parties using the civic voluntarism model
}

\begin{abstract}
Who are the people who can imagine joining a political party but do not act upon it? Based on representative data from the German General Social Survey (GGSS) of the years 2008 and 2018, this study analyzes factors that have an impact on wanting to work actively in a political party. The empirical analysis investigates to what extent socio-demographic resources, motivation, self-efficacy and involvement in social networks are related to the will to actively participate in parties. The logistic regression models show little influence of socio-demographic factors. However, political attitudes, such as trust in parties, are crucial explanatory factors. This is particularly the case in eastern German states. Overall, the results suggest that the model of civic voluntarism can be used at least partially for explaining individual preferences of joining a political party.
\end{abstract}

\section{Einleitung}

,... there is nothing wrong with those who do not participate, there is something unusual about those who do" schließt Fiorina (1999, S. 416) für die Mehrheit der Bürgerinnen und Bürger, die etwa aus Zufriedenheit oder Bequemlichkeit weitgehend auf politische Partizipation verzichten und sich bewusst entschieden haben, nicht politisch aktiv zu sein (Dahlgren 2009). Dieser Beitrag thematisiert mit der Mitgliedschaft in Parteien eine der Partizipationsformen, die in den meisten etablierten Parteien einem stetigen Niedergang entgegensieht (Niedermayer 2013). Generell wird hinterfragt, ob das Zeitalter der Massenparteien und Mitgliederparteien nicht längst vorbei ist und andere Partizipationsformen an ihre Stelle treten (Jun 2009b, 2011; Gibson et al. 2017). Die verschiedenen Teile von Parteien (party in central offices, party in public offices, party on the ground, Key 1953), Parteitypen und Parteienwandel werden viel beforscht ${ }^{1}$. Der Fokus liegt bis dato vor allem auf der Partei in öffentlichen Ämtern sowie den Parteizentralen (Bardi et al. 2014; Müller 2000). Dies ist deshalb von Belang, weil das Regieren und die Distribution von Ressourcen (Geld, Ämter, Mitglieder) zentral für die Persistenz von Parteien im System sind (Panebianco 1988).

Gerade jene Quelle, die Parteimitglieder darstellen, gerät aber oftmals in den Hintergrund der Forschung, wobei Mitglieder vor allem für große Parteien in demokratischen Systemen nach wie vor eine wichtige Basis zur Mobilisierung von Wählerinnen und Wählern darstellen (Whiteley 2011; Ware 1992) - als Multiplikatorinnen und Multiplikatoren auf der Straße. Geld und Ämter sind zwar numerisch und quantitativ einfacher zu erfassen, die Erfassung von Einstellungen und Orientierungen der Mitglieder gegenüber ihrer Partei dagegen geriert sich schwerer (Spier et al. 2012). Dies ist umso mehr der Fall, will man die Gruppe der Bevölkerung betrachten, die grundsätzlich einer Partei beitreten würde, dies aber (noch) nicht

\footnotetext{
1 Poguntke (2017); Katz und Mair (1994); Harmel und Janda (1994); Bardi et al. (2014); Fabre (2008); Deschouwer (1996); Mair et al. (2004); Wiesendahl (2010); Harfst et al. (2017).
} 
getan hat. Deshalb geht dieser Artikel folgender Frage nach: Was charakterisiert diejenigen Bürgerinnen und Bürger, die sich vorstellen, in einer Partei aktiv sein zu können, dies aber (noch) nicht getan haben? Zur Annäherung an diese Frage wird ermittelt, was die hier so genannten prinzipiell parteiaktivitätsbereiten Bürgerinnen und Bürger ausmacht, über welche Eigenschaften sie verfügen und ob es regionale Unterschiede innerhalb Deutschlands gibt. Wie ,tickt“ also das Reservoir derjenigen, die sich grundsätzlich vorstellen können, aktiv in einer Partei mitzuarbeiten, inwieweit entsprechen diese den Annahmen der Partizipationsforschung und was zeichnet sie womöglich aus?

Wir beziehen uns hierbei auf das Civic Voluntarism Model (CVM) nach Verba et al. (1995). Dieses bündelt die wichtigsten Faktoren der Mikro-Ebene zur Erklärung politischer Partizipation, wurde aber bis dato nicht substanziell erweitert (Gabriel 2012, 2018). Die verschiedenen Elemente des CVM werden hierzu einer Prüfung im Lichte der Parteimitgliederforschung unterzogen.

Hintergrund des Beitrags ist die Beobachtung rückläufiger Parteimitgliedschaften vor allem in den Großparteien SPD und CDU in Forschung, Medien, aber auch der Öffentlichkeit. Dieser Rückgang folgt seit den 1980er-Jahren einem stabilen Trend (Niedermayer 2016). Für einen Niedergang des Modells der Mitgliederpartei sprechen nicht nur die hohen Austrittszahlen. Auch die Überalterung der Parteimitglieder wird den Mitgliederschwund weiter beschleunigen (Dose und Fischer 2013). Beide Befunde beleuchten die sinkende Zahl der Parteimitgliedschaften jedoch nur von einer Seite: Demgegenüber stehen ausbleibende Eintritte, die sich nur in Wahljahren oder vor bundespolitisch wirkmächtigen und massenmedial kommunizierten innerparteilichen Beteiligungsevents wie der Abstimmung der SPD-Mitglieder über den Koalitionsvertrag 2013 und 2018 sprunghaft zum Positiven verändern. An dieser Stelle darf der Faktor Person in Bezug auf Parteibeitritte nicht unterschätzt werden: So konnte Jeremy Corbyn in Großbritannien einen massenhaften Anstieg der Mitgliedschaftszahlen bereits vor seiner erfolgreichen Wahl zum Parteivorsitzenden 2015 verbuchen (Poletti et al. 2016). Auch in Deutschland war die positive Wirkung eines Wechsels des Parteivorsitzes auf die Mitgliederbilanz einer Partei kurzfristig sichtbar: Die Übergabe des Parteivorsitzes von Sigmar Gabriel an Martin Schulz vor der Bundestagswahl 2017 führte zu einem wahren ,Hype“ unter SPD-Mitgliedern, die hiernach in sozialen Netzwerken mit einem neuen politischen Selbstwertgefühl auftraten. Unterdessen konnte die Partei von einem überdurchschnittlichen Anstieg von Neumitgliedern profitieren (Niedermayer 2017); so seien alleine 17.000 Neumitglieder in den ersten vier Monaten nach der angekündigten Kanzlerkandidatur von Martin Schulz in die SPD eingetreten (Grunden et al. 2017).

Wiesendahl wies bereits 2011 darauf hin, dass sich etwa $15 \%$ der Bundesbürgerinnen und Bundesbürger grundsätzlich vorstellen können, einer Partei beizutreten bzw. aktiv in ihr mitzuarbeiten. Diese Zahlen, die den Daten der Allgemeinen Bevölkerungsumfrage der Sozialwissenschaften (ALLBUS) des Jahres 2008 entsprechen, sind jedoch zu hoch veranschlagt, da bereits organisierte Parteimitglieder darunter gefasst werden. Der bereinigte Wert beträgt in diesem Falle knapp zwölf Prozent, womit sich dennoch konstatieren lässt, dass ein nicht zu unterschätzendes Reservoir an Bürgerinnen und Bürgern in der deutschen Bevölkerung existiert, die aktiv in Parteien mitarbeiten würden. Dass die Parteien versucht haben, dieses Reservoir zu 
aktivieren, lässt sich an den verschiedenen neuen Mitgliedschaftsformen erkennen, die in den letzten Jahren eingeführt bzw. erprobt wurden - jedoch mit begrenztem Erfolg (Jun 2009b; Gerl et al. 2016). Es ist den deutschen Parteien mehrheitlich (mit Ausnahme der Grünen sowie neuen Parteien in den ersten Jahren ihrer Existenz wie bspw. den Piraten oder der AfD) offensichtlich nicht gelungen, mithilfe dieser neuen Mitgliedschaftsformen auch neue Mitglieder zu rekrutieren. Scarrow (2014) schlägt angesichts dieser Entwicklung, die auch auf internationaler Ebene zu beobachten ist (van Biezen et al. 2012; Gauja 2017), vor, dass Parteien drei wesentliche Strategien zur Rekrutierung von Neumitgliedern verfolgen sollten: erstens eine Stärkung der klassischen Mitgliedschaft, zweitens eine Reduktion der Beitrittskosten sowie drittens eine Neudefinition dessen, was ein Parteibeitritt bedeutet und an Verpflichtungen nach sich zieht (Scarrow 2014). Die letzte Strategie verlagert den Fokus von einer formalen Mitgliedschaft auf ein unverbindliches Unterstützermodell, wie es einige deutsche Parteien bereits kennen - etwa die SPD (§ 10a Organisationsstatut der SPD). Damit verbunden ist letztlich auch ein mögliches Aufweichen der Mitgliedschaft, was dazu führt, dass die Grenzen der Parteien durchlässiger und fluider werden. Ohne fluide Formen von Mitgliedschaft im Sinne des genannten Unterstützermodells und einer Öffnung von Parteien in diese Richtung geht aktive Mitarbeit ernsthaft nur im Rahmen einer Mitgliedschaft. Diese muss aber deutlich von der Absichtsbekundung, aktiv in einer Partei mitarbeiten zu wollen, unterschieden werden.

Wenngleich die deutschen Großparteien einzelne der von Scarrow (2014) genannten Strategien ausprobiert haben, so gelang es ihnen bisher nicht, den Trend der sinkenden Mitgliedschaft umzukehren oder aufzuhalten. Die Parteienforschung untersucht diesbezüglich, warum bereits bestehende Mitglieder einer Partei beigetreten sind, warum sie ausgetreten sind und wie Parteien neue Mitglieder an sich binden können (Spier et al. 2012). Sie ignoriert jedoch den Hinweis von Wiesendahl (2011), den Blick auf potenzielle, noch nicht beigetretene Personen zu richten. So liegt ein logischer Fehlschluss vor, wenn von den Mitgliedern auf die Motivationen der Bürgerinnen und Bürger geschlossen wird, die sich eine Mitgliedschaft bzw. eine Aktivität in Parteien lediglich vorstellen können. Bezüglich der Anreizstrukturen und der Frage nach dem Beweggrund zum Parteibeitritt zeigen Poletti et al. (2018) relevante Ergebnisse hinsichtlich der erwarteten und tatsächlichen Beitrittsmotive auf. Sie stellen fest, dass Unterstützerinnen und Unterstützer andere Beitrittsmotive bei Parteimitgliedern annehmen (soziale Normen und persönliche Vorteile) als die tatsächlichen Beitrittsgründe (ideologische Gründe, politische Themen, Führungspersonen). Gerade zu dieser Gruppe fehlt es jedoch bisher an weiterer Forschung. Daher möchte dieser Artikel dazu beitragen, die genannte Forschungslücke zu schließen, indem zunächst auf Theorien der Mobilisierungsforschung wie das CVM eingegangen wird, wobei Anreize und Hindernisse für aktive Parteiarbeit diskutiert werden. Anschließend werden aus diesen Überlegungen Hypothesen abgeleitet, die empirisch anhand der ALLBUS-Daten von 2008 und 2018 überprüft werden. Abschließend werden die empirischen Ergebnisse in den bestehenden Forschungskontext eingeordnet und mit Blick auf zukünftige Forschungsrichtungen diskutiert. 


\section{Politische Partizipation zwischen „Können, Wollen und Gefragt Werden"}

\subsection{Die Voraussetzungen für aktive Mitarbeit in Parteien als Vorform für eine Parteimitgliedschaft}

Etablierte Parteien leiden, so konstatiert es die Parteienforschung unentwegt, gleich unter mehreren gesellschaftlichen Wandlungsprozessen, die die Nachfrage nach einer Parteimitgliedschaft negativ beeinflussen. Zur Beantwortung der hier zugrundeliegenden Fragestellung nach dem vorhandenen Reservoir potenziell Beitrittsbereiter ist es nicht notwendig, die Debatten über party change und party decline umfassend zu erläutern (ausführlicher: Decker 2015; Holtmann 2012). Festzuhalten ist: Die Parteien haben durch die sinkenden Mitgliederzahlen ihre Rolle als Fürsprecher ideologisch orientierter Gesellschaftsgruppen (Arbeiterinnen und Arbeiter für die SPD, Mittelständlerinnen und Mittelständler sowie Selbständige für die FDP, Unternehmerinnen und Unternehmer für die CDU usw.) verloren. Mitgliederparteien sehen sich aufbrechenden Milieus entlang klassischer Konfliktlinien gegenübergestellt (Lipset und Rokkan 1967; Eith und Mielke 2017). Die sinkende gesellschaftliche Anbindung führte zu einer verminderten Identifikationskraft zur Ausschöpfung des ehemals vorhandenen Mobilisierungspotentials - und dies unterschiedlich in Westund Ostdeutschland (Niedermayer 2013). Zugleich haben sich die Großparteien der Entwicklung aufbrechender Milieus angepasst und sich von der Massenintegrationspartei zur Catch-All-Party gewandelt (Kirchheimer 1965; Katz und Mair 1994). Damit hat sich jedoch eine Verschiebung in der Rekrutierung der Neumitglieder ergeben, da Parteimitglieder zumeist aus den parteinahen Milieus und nicht aus der Gesamtgesellschaft stammen (Jun 2009a, S. 237).

Zugleich ist nicht zuletzt auch seit der,Wiedervereinigung ' eine fortgesetzte Individualisierung der Gesellschaft festzustellen (Kornelius und Roth 2004). Dieser „Individualisierungsschub“ (Schimank 2012, S. 31) fand auch parteiendemokratisch mit der Gründung der Grünen seinen ,parlamentarischen Ausdruck“ (Schimank 2012, S. 31). Zudem stieg das politische Wissen der Bürgerinnen und Bürger sowie das kritische Hinterfragen der Großparteien an (Geißler 2014), was nicht zuletzt im Sprachduktus widergespielt wird, wenn zwischen, etablierten ' und ,nicht-etablierten " Parteien unterschieden wird. Hinzu kommt ein konstant niedriges Vertrauen in Parteien (Biehl 2013), das im Wechselspiel mit den anderen genannten Faktoren eine Parteimitgliedschaft immer unwahrscheinlicher macht. Zwar wird auch anderen intermediären Organisationen allgemeinhin nur wenig Vertrauen entgegengebracht, allerdings wirkt es sich für Parteien besonders schwerwiegend aus. Das ist insofern problematisch, als Parteien als zentrales Bindeglied zwischen Staat und Gesellschaft fungieren (sie treten zu Wahlen an und stellen Vertreterinnen sowie Vertreter für politische Ämter; Sartori 2005).

Überdies sind Bürgerinnen und Bürger inzwischen weniger bereit, sich langfristig an eine Partei zu binden (Scarrow 2014). Wenngleich die Parteien keine langfristige Bindung erzwingen, so wirkt die Mitgliedschaft in einer Großorganisation vermeintlich verbindlich und gewissermaßen unumkehrbar. Auch ist die Parteimitgliedschaft recht voraussetzungsreich: Das Ausfüllen und Unterzeichnen eines Mitgliedsantra- 
ges ist ebenso wie das Zahlen eines Mitgliedsbeitrages obligatorisch, weil parteienrechtlich vorgeschrieben. Der Parteibeitritt bzw. die Parteimitgliedschaft setzen zuvorderst das Vorhandensein der Ressource ,Geld“ voraus, die sowohl im sozioökonomischen Standardmodell politischer Partizipation als auch im noch zu erläuternden CVM eine tragende Rolle spielt. Zugleich setzt die Parteimitgliedschaft voraus, diese Ressource entbehren zu wollen, ohne eine direkte Gegenleistung dafür zu erhalten. Die Teilnahme an Parteiveranstaltungen ist zudem zeitintensiv, was den hohen Anteil inaktiver Mitglieder in den deutschen Parteien erklärt (überhaupt nicht aktiv sind $31 \%$ bei der CDU im Jahr 2009 und 33\% bei der SPD im Jahr 2009; Spier 2012; Korte et al. 2018).

Erfragt man die Ursache für Parteibeitritte, so geben immerhin elf Prozent der Parteimitglieder in der deutschen Parteimitgliederstudie an, dass eine bestimmte Politikerin oder ein bestimmter Politiker für sie den konkreten Anlass zum Parteibeitritt bot (Laux 2011), was auf die Wichtigkeit des bereits erwähnten Faktors Person hinweist. Andere Anlässe zum Beitritt wie Protest, soziale Beziehungen, gesellschaftliche bzw. politische Ereignisse sowie politisches Interesse und ein Wunsch nach Einfluss rangieren quantitativ jedoch noch über dem politischen Personal als Eintrittsgrund in eine Partei.

Zuletzt ist speziell für den deutschen Fall auf die ehemalige Teilung in Ostund Westdeutschland hinzuweisen. Hierbei ist zum einen $\mathrm{zu}$ beachten, dass die westdeutschen Parteien in Ostdeutschland erst ab 1990 überhaupt eine Bindung zu den Wählerinnen und Wählern aufbauen konnten, da sie vorher schlichtweg nicht existent waren (Schoen und Weins 2014). Zum anderen hatten es Parteien wie die Grünen und die FDP äußerst schwer, Wählerinnen und Wähler in Deutschland zu erreichen - PDS bzw. Linkspartei hingegen entwickelten sich zur veritablen Ostpartei (siehe dazu auch Arzheimer und Falter 2013, S. 132). Für die Parteibeitrittsbereitschaft und diese Untersuchung bedeutet dies aber letztlich nur eine konzeptionelle Verschiebung zwischen den Parteien, zu denen ein Beitritt gesucht wird, will man von Wahlverhalten auf Partizipationsverhalten für Parteien schließen. Werden die Ergebnisse von Holtmann und Kolleginnen und Kollegen betrachtet, so scheinen sich regionale Unterschiede zwischen Ost und West hinsichtlich konventioneller Partizipation zu relativieren, ein Befund, der überraschend scheint: ,Wie unsere Bevölkerungsumfrage 2018 zeigt, lassen sich knapp 30 Jahre nach der deutschen Wiedervereinigung - mit der Ausnahme des Wahlverhaltens - keine großen Unterschiede in der aktiven Beteiligung der West- und der Ostdeutschen an Politik ausmachen“ (Holtmann 2020a, S. 329). Eine Abschwächung der Unterschiede selbst beim von Holtmann hervorgehobenen Wahlverhalten zeigt Arzheimer (2016), wohingegen diese Unterschiede bei Arzheimer und Falter (2013, S. 145) noch deutlich sichtbar waren. Schmitt-Beck und Weick (2001, S. 4) zeigen, dass in den ersten Jahren nach der deutschen Wiedervereinigung geringere Bindungen an Parteien im Osten gegenüber dem Westen beobachtbar waren. Zwar haben sich auch diese mittlerweile weitgehend angeglichen (Jaeck 2020, S. 48), doch ist die Frage zu stellen, ob Parteiidentifikation allein genügt, um auch parteiaktivitätsbereit zu sein oder ob nicht vielleicht eher Faktoren wie das Vertrauen in Parteien dafür ausschlaggebend sind. Die Ausgangslage der Forschung bietet damit ein für die vorliegende Untersuchung weiterhin unklares Bild: Wenngleich sich die Unterschiede zwischen Ost und West 
bei konventioneller Partizipation anzugleichen scheinen, so sind dennoch maßgebliche Unterschiede im Vertrauen zu Parteien festzustellen; das Vertrauen in Parteien ist zwar sowohl in West- als auch in Ostdeutschland insgesamt recht niedrig, in Ostdeutschland aber weiterhin noch niedriger ausgeprägt (Jaeck 2020, S. 45). Damit kann gerade nicht angenommen werden, dass sich die Parteiaktivitätsbereitschaft als eine Art der Vorform oder Prämisse zum Parteibeitritt zwischen Ost und West analog zu den vorgenannten Partizipationsformen - angeglichen haben könnte.

Da sowohl konzeptionelle Bedenken vorliegen als auch empirische Untersuchungen zum Forschungsgegenstand dieses Beitrags fehlen, wird diese Untersuchung ermitteln, welche Unterschiede für die Parteiaktivitätsbereitschaft zwischen Westund Ostdeutschland festzustellen sind.

\subsection{Das Civic Voluntarism Model}

Das Civic Voluntarism Model liefert den wohl umfassendsten Versuch, politische Partizipation auf der Mikro-Ebene zu erfassen (Verba et al. 1995). Der Ausganspunkt liegt in der Erklärung der politischen Nicht-Beteiligung. ,[P] eople may be inactive because they lack resources, because they lack psychological engagement with politics, or because they are outside of the recruitment networks that bring people into politics“ [Hervorhebungen i. O.] (Verba et al. 1995, S. 16). Verfügt eine Bürgerin oder ein Bürger über Ressourcen, Motivation und entsprechende soziale Netzwerke bedeutet dies lediglich, dass die Wahrscheinlichkeit zu partizipieren gesteigert ist, nicht aber, dass Partizipation unweigerlich erfolgt. Soziale Netzwerke werden von den Autoren recht vage definiert und umfassen die Familie und Freunde, aber auch Kontakte zu anderen Menschen in der Schule, am Arbeitsplatz, in Freiwilligenorganisationen oder in Kirchen (bzw. religiösen Organisationen allgemein) (Schlozman et al. 2013) - sprich, diverse soziale Kontakte.

Diesen Faktoren vorgelagert steht das legale Recht, der eigenen Stimme Gehör verschaffen zu können bzw. zu dürfen (Schlozman et al. 2013). Für den Parteibeitritt bedeutet dies, kleinere Einschränkungen vorzunehmen. So ist ein bestimmtes Alter, das die Parteien selbst festlegen (Bukow 2013), Beitrittsvoraussetzung. Auch ist die gleichzeitige Mitgliedschaft in anderen Parteien zumeist verwehrt (lediglich die Piraten kennen eine solche Ausnahme). Ist der Beitritt rechtlich möglich, werden die folgenden von Verba et al. (1995) genannten Faktoren zur Erklärung politischer Beteiligung relevant. Unter den Ressourcen [resources] fassen die Autoren Fähigkeiten [skills], Wissen, Zeit und Geld zusammen. Sie stellen fest, dass diejenigen Bürgerinnen und Bürger, die über eine höhere Bildung verfügen, auch eher in der Lage sind, politisch zu partizipieren. Sie können durch ihr Wissen leichter an Informationen gelangen und diese besser verarbeiten (Schlozman et al. 2013). Dies wiederum befähigt sie dazu, selbst politisch teilzuhaben. Diese Ressourcen allein sind aber, dem Modell folgend, nicht ausreichend, um das Warum der Partizipation zu erklären. Ressourcen, die in das Socioeconomic Status Model (im Folgenden SES-Modell) eingeflossen sind, wurden zwar lange Zeit zur Erklärung politischer Partizipation herangezogen (Barnes et al. 1979; Morales 2009), doch konnten sie nicht erklären, warum ein Mensch, der reicher oder gebildeter ist, sich häufiger politisch beteiligt. 
Die Autoren fügen daher den Faktor der Motivation hinzu. Zur Motivation gehören politisches Interesse sowie politische efficacy, also ein Empfinden politischer Selbstwirksamkeit (Schlozman et al. 2013). In der efficacy spiegelt sich nicht nur die selbst wahrgenommene Fähigkeit wider, politische Entscheidungen beeinflussen zu können, sondern der prinzipielle Wille, dies zu tun. Mit der Unterscheidung von internal und external efficacy bietet die Forschung zur Selbstwirksamkeit zwei Konstrukte an, die essenziell für politische Beteiligung sind und zudem das Gefühl der Partizipierenden beinhalten, dass das politische System sowie die politischen $\mathrm{Au}$ toritäten sich überhaupt um die Gedanken der Bürgerinnen und Bürger ,kümmern * (Vetter 2000) - also responsiv sind. Internal efficacy meint dabei das ,politische Kompetenzbewusstsein“, external efficacy „das Gefühl politischer Responsivität“ (Falter und Schoen 2014, S. 867), was in einem gewissen Maße notwendig ist, um einer politischen Organisation beizutreten (Morales 2005).

Die sozialen Netzwerke sind als dritter Faktor der Partizipation zu beachten. Sie lösen laut Verba et al. (1995) Partizipation im letzten Schritt aus. Die Erklärung dafür lautet, dass einige Menschen trotz vorhandener Ressourcen sowie Motivation noch eine Art ,sozialen Aufruf' benötigen, um an politischen Aktivitäten teilzunehmen. So zeigt sich in den Studien der Autoren, dass vor allem Freunde (im Gegensatz zu Fremden) die stärkste Rekrutierungswirkung entfalten können, weil hier ein Bedürfnis nach sozialer Erwünschtheit geweckt wird (Verba et al. 1995). In ihren persönlichen und beruflichen Netzwerken sprechen Menschen unter anderem über Politik und tauschen sich zu politischen Themen aus. Zudem können politisch bereits engagierte Bürgerinnen und Bürger im eigenen Netzwerk auf Möglichkeiten zur politischen Beteiligung hinweisen. Dies kann den letzten und zugleich notwendigen Schritt darstellen, der motivierte und mit den notwendigen sozioökonomischen Ressourcen ausgestattete Bürgerinnen und Bürger zu politischer Beteiligung bringt.

Auch empirisch wirkt das Modell, blickt man auf die unterschiedlichen Befunde aus der Literatur: Bürgerinnen und Bürger mit höheren formalen Bildungsabschlüssen partizipieren über alle Partizipationsarten hinweg häufiger als geringer gebildete Bürgerinnen und Bürger (Mays und Hambauer 2016), was die Bildung zu einem starken Prädiktor für politische Partizipation macht. Allerdings werden gerade die exakten Kausalmechanismen, die von der Bildung auf die Partizipation wirken, noch erforscht (Persson 2015). Neben dem Faktor Bildung hat sich Zeit als Ressource herauskristallisiert, die Bürgerinnen und Bürgern bleiben muss, um in ihrer Freizeit politischen Aktivitäten nachgehen zu können (Mays und Hambauer 2016). Fehlt diese, ist politische Partizipation in Parteien und anderen politischen Organisationen eher unwahrscheinlich.

Auch die beruflichen Aktivitäten können innerhalb des Modells eine partizipationsfördernde Rolle einnehmen: Vielfältigere Anforderungen im Beruf sowie längere Arbeitszeiten haben diverse Wirkungen auf politische Partizipation. Berufe, die positiv mit politischer Partizipation korrelieren, sind mit (hoher) Bildung und einem „on-the-job training“ (Schlozman et al. 1999, S. 37) verbunden. Vor allem höhere berufliche Positionen verstärken Partizipation - im Gegensatz zu eher niedrigen beruflichen Positionen (Schlozman et al. 1999). Partizipationsfördernd wirken Berufe und berufliche Netzwerke vor allem dann, wenn in ihnen Fähigkeiten ausgebildet werden, die auch der politischen Partizipation dienen, beispielsweise das Schreiben 
von Briefen, die Organisation von Arbeitstreffen und Meetings sowie die Ausbildung und Nutzung rhetorischer Fähigkeiten (Verba et al. 1995).

Wie die einzelnen Faktoren des CVM zusammenwirken und wann Motivation in einem ausreichenden Maß vorhanden ist, um zu politischer Beteiligung zu führen, unterscheidet sich individuell. Die vielschichtige Natur von Motivation (Hooghe 2014) erlaubt es ebenfalls nicht, einen spezifischen Motivator herauszustellen. Zur Wirkung sozialer Netzwerke ist auf Basis empirischer Beobachtungen hinzuzufügen, dass die Bindung zu Großorganisationen wie Kirchen und Gewerkschaften seit Jahren abnimmt (Ebbinghaus und Göbel 2014). Gerade diese Großorganisationen sind aber zumindest strukturell mit Parteien vergleichbar, insbesondere in den Hierarchiestrukturen sowie dem organisationalen Aufbau. Mit der rückläufigen Gewerkschaftsmitgliedschaft ist ein für Parteien maßgebliches, weil ihnen potenziell nahestehendes Netzwerk ebenfalls im Schwinden begriffen. So ist auf Basis des CVM anzunehmen, dass solche Netzwerke zur Parteiaktivität anhalten können, aber freilich nur dann, wenn sie selbst überhaupt noch über genügend Mitglieder verfügen. Dass das Ausdünnen dieser Netzwerke beispielsweise bei der aktiven Parteimitgliederwerbung zum Problem werden kann, zeigen gegenwärtige empirische Befunde unter Mitgliederwerberinnen und -werbern von SPD und CDU (Jakobs 2021).

Doch auch die Mitgliedschaft in nicht-politischen Freiwilligenorganisationen kann partizipationsfördernd wirken (Acik 2013), da Bürgerinnen und Bürger dort zum einen ihre bürgerlichen Fähigkeiten (civic skills) erweitern und zum anderen in Netzwerke integriert werden, die Engagement per se fördern. Diesen Zusammenhang erkannten bereits Almond und Verba (1989 [1963]) und Verba und Nie (1972), die ihn empirisch untersuchten. In der Forschung wird der Konnex zwischen Netzwerken und politischem Engagement jedoch in Teilen angezweifelt: So zeigen Montero et al. (2007) zwar ebenfalls auf, dass politische Partizipation und die Einbindung in soziale Netzwerke miteinander verknüpft sind, doch vermuten die Autoren dahinter keinen Kausalzusammenhang, sondern eine Korrelation, die auf gemeinsame Ursachen zurückzuführen sei.

Netzwerke wirken sich dessen ungeachtet nicht immer ausschließlich positiv auf politische Beteiligung aus. Sie können politische Partizipation auch gänzlich hemmen. Ist das soziale Netzwerk einer Bürgerin oder eines Bürgers unpolitisch oder gar politikfeindlich eingestellt, kann dies eine durchaus partizipationshemmende Wirkung entfalten. Diesem Ansatz zufolge kann die eigene Parteibeitrittsbereitschaft in der bloßen Intendierungsphase verharren, und zwar aufgrund der Ablehnung von Parteien oder Abneigung ihnen gegenüber, die Individuen aus ihren sozialen Netzwerken erfahren, was wiederum die Verhaltensausübung blockiert - trotz vorhandener Ressourcenausstattung und potenzieller Motivation.

Aus den vorangegangenen Überlegungen lassen sich mehrere Hypothesen zur empirischen Überprüfung ableiten. Basierend auf den Annahmen des SES sowie des CVM ist in einer ersten Hypothese davon auszugehen, dass das Vorhandensein eines hohen Bildungsstandes sowie der Ressourcen Zeit und Geld die Bereitschaft zur Mitarbeit in einer Partei fördern. Zeit und Geld müssen dabei jedoch in Kombination betrachtet werden: So bringt Geld allein noch keine verfügbare Zeit zur Partizipation und umgekehrt geht viel verfügbare Zeit nicht unbedingt mit einem hohen Maß 
an Partizipation einher. ${ }^{2}$ Daraus lassen sich folgende erste Hypothesen bilden, die miteinander verknüpft sind:

H1a Ein hoher sozioökonomischer Status führt zu einer gesteigerten Bereitschaft, in einer Partei aktiv mitarbeiten zu wollen.

H1b Viel Zeit führt zu einer gesteigerten Bereitschaft, in einer Partei aktiv mitarbeiten zu wollen.

Neben den Ressourcen ist im CVM jedoch auch die Motivation zu berücksichtigen; ein Verharren in der Ressourcenperspektive allein wäre konzeptionell unterkomplex. Insbesondere das politische Wirksamkeitsgefühl dürfte eine große Rolle dabei spielen, selbst politisch aktiv werden zu wollen. Ein hohes Maß an efficacy müsste den vorangegangenen Überlegungen dieses Artikels zufolge zu einer höheren Motivation und damit zu einer erhöhten Parteiaktivitätsbereitschaft führen. Hinzu tritt das politische Interesse, das sich als eine Art chronologische Vorstufe zur efficacy betrachten lässt: Sind Bürgerinnen und Bürger politisch interessiert, kann dies die Bereitschaft zur politischen Teilhabe konstituieren, wobei die efficacy als weiterer motivierender Faktor hinzutritt. Ein zweites Hypothesenbündel für die empirische Untersuchung ist daher:

H2a Ein hohes Maß an politischer efficacy führt zu einer gesteigerten Motivation, in einer Partei aktiv mitarbeiten zu wollen.

H2b Ein hohes Maß an politischem Interesse führt zu einer gesteigerten Motivation, in einer Partei aktiv mitarbeiten zu wollen.

Das Postulat des CVM, dass Menschen zu politischer Partizipation durch starke Einbindung in soziale und berufliche Netzwerke getriggert werden, kann die Leerstelle der Parteimitgliederforschung füllen, wenn Ressourcen und Motivation allein eben nicht in einen Parteibeitritt münden. Ausgehend von den Annahmen des CVM sollten Bürgerinnen und Bürger, die stark in soziale Netzwerke eingebunden sind, partizipationsbereiter sein als solche, bei denen diese Bindung nicht vorliegt. Die dritte Hypothese lautet daher:

H3 Eine starke Eingebundenheit in berufliche und soziale Netzwerke führt zu einer gesteigerten Bereitschaft, in einer Partei aktiv mitarbeiten zu wollen.

Nicht zuletzt bilden Parteien für die Partizipationsforschung einen gewissen Sonderfall. Stetig abnehmende Mitgliederzahlen, niedriges Vertrauen in Parteien und eine sinkende Parteiidentifikation bilden allesamt Indikatoren, die aus der Parteiaktivitätsbereitschaft eine besondere Form politischer Partizipation konstruieren. Es wird daher angenommen, dass die Bereitschaft, aktiv in einer Partei mitzuarbeiten, maßgeblich von einer positiven allgemeinen Evaluation von Parteien, und zwar vor allem durch Vertrauen in diese, abhängt. Hieraus geht die vierte und letzte Hypothese hervor:

\footnotetext{
2 Zeit wird im hier vorliegenden Fall über die Variablen Zeitaufwand für Freunde/Kollegen/Vereinskollegen sowie Vereinstätigkeit abgebildet (s. Tab. 4, 5 und 6 im Anhang).
} 
H4 Ein hohes Maß an politischem Vertrauen in politische Parteien führt zu einer gesteigerten Bereitschaft, in einer Partei aktiv mitarbeiten zu wollen.

\section{Methoden und Daten}

Neben den bereits genannten ALLBUS-Daten gibt die deutsche Parteimitgliederstudie von 2009 einen dazu ähnlichen Wert von 16\% von Bürgerinnen und Bürgern an, die prinzipiell parteibeitrittsbereit sind (Rohrbach 2013). Um die Gründe der Beitrittsbereitschaft zu ergründen, stützt sich die folgende Analyse auf ALLBUS-Daten, die das Item zur Mitarbeitsbereitschaft zuletzt 2008 sowie 2018 enthielten. Beide Datensätze beinhalten Fragen dazu, inwiefern die Bundesbürgerinnen und Bundesbürger zur Mitarbeit in Parteien bereit sind, was über ein Item im Fragebogen abgefragt wurde und insofern einen Näherungswert für die potenzielle Parteimitgliedschaft widergibt. ${ }^{3}$ Durch das Sampling-Design des ALLBUS ist es zudem möglich, für Ost- und Westdeutschland repräsentative Ergebnisse mittels eines Subgruppenvergleichs zu erzielen, sodass die Beitrittsbereitschaft in beiden Gebieten analysiert werden kann. Insofern unternehmen wir nicht nur einen Zeitvergleich zwischen den beiden Messzeitpunkten, sondern tragen auch zur Literatur zu verschiedenen Partizipationskulturen in Ost und West bei. Gerade für die Parteiaktivitätsbereitschaft ist die Untersuchung eventuell vorhandener Ost-West-Unterschiede in diesem Beitrag nicht nur präzedenzlos, sondern auch als Ergänzung zu aktuellen Befunden (bspw. Holtmann 2020b) zu betrachten, die sich mit Ost-West-Unterschieden im Partizipationsverhalten der deutschen Bevölkerung beschäftigen.

Die Parteiaktivitätsbereitschaft wurde als eine mögliche Antwort auf eine Multiple-Choice-Frage mit Mehrfachnennungen erfasst (,In einer Partei aktiv mitarbeiten"). Die potenziell aktive Mitarbeit in Parteien ist jedoch nicht mit dem unmittelbaren Wunsch zum Beitritt gleichzusetzen. Dennoch kann über dieses Item die prinzipielle Nähe zu einer Partei jenseits der klassischen Items zur Parteiidentifikation abgefragt werden. Befragte, die bekunden, aktiv in einer Partei mitarbeiten zu wollen, um sich politisch in einer Sache einbringen zu wollen, scheinen dem organisatorischen Aufbau und der Arbeitsweise von Parteien grundsätzlich zugeneigt zu sein und sehen diese - so die Annahme - als Vehikel zur Beförderung eigener politischer Anliegen. Zudem ist zu erwarten, dass bei einer tatsächlichen aktiven Mitarbeit der Beitritt naheliegt oder in Zukunft voraussichtlich erfolgen wird.

Als unabhängige Variablen zur Erklärung der intendierten Parteimitarbeit wurden in Bezug auf die Hypothesen die folgenden herangezogen (vgl. Tab. 6 im Anhang). Die Hypothese 1a wurde über sozioökonomische Faktoren wie Alter (in Jahren), Geschlecht, Bildungsstand anhand des Schulabschlusses (niedrig, mittel, hoch), Ein-

\footnotetext{
3 Die Frage im ALLBUS-Fragebogen lautete ,Wenn Sie politisch in einer Sache, die Ihnen wichtig ist, Einfluss nehmen, Ihren Standpunkt zur Geltung bringen wollten: Welche der Möglichkeiten auf diesen Karten würden Sie dann nutzen, was davon käme für Sie in Frage?“ (Terwey und Baltzer 2011, S. 37). Dabei war ,in einer Partei mitarbeiten“ eine mögliche Antwortkategorie (E) innerhalb verschiedener Beteiligungsformen (vgl. GESIS 2015, 2018, S. 10) Zudem beinhaltet dieser Wert in den Datensätzen auch all jene Bürgerinnen und Bürger, die bereits Mitglied einer Partei sind. Diese Variablenausprägung soll hier jedoch nicht mit der Bereitschaft, tatsächlich beizutreten gleichgesetzt werden.
} 
kommen (Nettoäquivalenzeinkommen) sowie die Beschäftigungsart (Vollzeit, Teilzeit, arbeitslos) operationalisiert. Die Hypothese 1b wurde über Vereinsmitgliedschaft (Tätigkeit in Vereinen), Zeitaufwand für Vereins- und Arbeitskollegen sowie Zeitaufwand für Freunde operationalisiert. Die Hypothesen $2 a$ und $2 b$ wurden über die politische efficacy (2a) und politisches Interesse (2b) operationalisiert: Das wurde auf zweierlei Art gemessen. Das politische Interesse wird anhand eines einzelnen Indikators basierend auf einer 5er-Likert-Skala gemessen, während politische Efficacy über zwei Mittelwertindizes aus je zwei Items als internal und external efficacy in die Modelle aufgenommen wird. Die Indizes zeigen dabei unter Ausschluss der Parteimitglieder eine akzeptable Reliabilität für external efficacy (2008: alpha =0,50; 2018: alpha=0,61) und internal efficacy (2008: 0,75; 2018: alpha=0,72). Um die Hypothese $3 \mathrm{zu}$ testen, werden der berichtete Zeitaufwand für Freizeitaktivitäten mit Mitmenschen (Kolleginnen und Kollegen, Freundinnen und Freunden, Familie) und der Mitgliedstatus in zivilgesellschaftlichen Organisationen als dichotome Summenindizes aufgenommen. Um die Hypothese $4 \mathrm{zu}$ testen, wird ein einzelner Indikator zum berichteten politischen Vertrauen in Parteien berücksichtigt (siehe zu deskriptiven Beschreibungen der einzelnen Variablen die Tab. 4 und 5 im Anhang).

Ausgehend von den theoretischen Überlegungen und Annahmen werden im Folgenden die vorgestellten Hypothesen mit binomialen logistischen Regressionsanalysen untersucht. Die Parteimitglieder wurden dazu aus der Analyse ausgeschlossen, um die Hypothesen zu untersuchen. Obschon ein Vergleich zwischen Parteimitgliedern, Beitrittsbereiten und den Nicht-Beitrittsbereiten wünschenswert wäre, reicht die Fallzahl der Parteimitglieder (2008: $N=101$; 2018: $N=121$ ) nicht aus, um multiple Regressionen mit einer Vielzahl an kategoriellen Erklärungsfaktoren wie Geschlecht, Erhebungsgebiet, Berufstätigkeit ${ }^{4}$ und Schulabschluss, wie es die theoretischen Annahmen erfordern, valide durchzuführen.

\section{Empirische Analyse}

In einem ersten Schritt wurden zunächst verschiedene Variablen mit deskriptiven Statistiken im Hinblick auf erste Eindrücke zu den Hypothesen überprüft (vgl. Tab. 4 und 5 im Anhang). Regressionsanalysen ermöglichen zu ermitteln, welche Merkmale den Parteiaktivitätsbereiten fehlen, die wiederum eine mögliche Erklärung zu ihrem Nichtbeitritt liefern. So sind deutliche Unterschiede zwischen West- und Ostdeutschland sowie zwischen den beiden Messzeitpunkten festzustellen: Tab. 1 zeigt, dass sich die Parteiaktivitätsbereiten in den jeweiligen bundesdeutschen Gebieten deutlich unterscheiden. Insgesamt beobachten wir im Gesamtsample einen Anstieg der potenziell Aktiven von 12,4 auf 19,5\% zwischen 2008 und 2018 - nach Ausschluss der aktiven Parteimitglieder. Bemerkenswert ist, dass die Parteiaktivitätsbereiten vornehmlich in Westdeutschland (21,5\%) leben. Auf die Gründe für

\footnotetext{
${ }^{4}$ Der Einbezug des Treiman-Prestiges (I68 oder ISOPS) wäre interessant gewesen. Da es aber nur auf Vollzeitbeschäftigte angewendet wird, war die Erklärkraft dieser Variablen nach einer drastischen Reduktion der Fallzahl um rund 1300 bzw. 1700 Befragte nicht hilfreich.
} 
Tab. 1 Bereitschaft zur aktiven Mitarbeit in einer Partei in Ostund Westdeutschland. (Quelle: Eigene Berechnung basierend auf dem ALLBUS 2008 und 2018)

\begin{tabular}{lll}
\hline & 2008 & 2018 \\
\hline Gesamtsample & 12,4 & 19,5 \\
Ost & 8,9 & 10,0 \\
West & 13,2 & 21,5 \\
\hline
\end{tabular}

Die berichteten Werte sind gültige prozentuale Anteile der beitrittswilligen Personen nach Ausschluss der bereits aktiven Parteimitglieder und im Falle des Gesamtsamples gewichtet

eventuelle regionale Unterschiede in diesem Beitrag nicht einzugehen, wäre also nachlässig.

Anhand von Mittelwertvergleichen zwischen Beitrittsbereiten, Nicht-Beitrittsbereiten und Parteimitgliedern für metrische Variablen und Tests auf statistische Unabhängigkeit für kategorielle Variablen wurde ermittelt, dass die Parteiaktivitätsbereiten sich von den Parteimitgliedern folgendermaßen signifikant unterscheiden $(p<0,001)$ : Sie verfügen über ein niedrigeres Einkommen, verfügen in allen verwendeten Items über eine geringere internal und external efficacy und sind weniger in Vereinen aktiv; zudem ist der Zeitaufwand, den sie für Vereinskolleginnen und Vereinskollegen sowie Arbeitskolleginnen und Arbeitskollegen aufwenden, geringer als derjenige, den Parteimitglieder aufwenden (vgl. Tab. 5 im Anhang).

Mithilfe von binomialen logistischen Regressionsmodellen ${ }^{5}$ (siehe Tab. 2 und 3) wird ermittelt, ob sich diese Unterschiede hinsichtlich der aufgestellten Hypothesen im Zeitvergleich und zwischen den Wohngebieten wiederfinden lassen, was die prinzipielle Bereitschaft zur Parteimitarbeit erklären könnte. Die Ergebnisse der Analyse für das gesamte Sample (nach Ausschluss der Parteimitglieder) sowie aufgeteilt nach Ost- und Westdeutschland, sind in Tab. 2 und 3 ersichtlich. Aufgrund der deutlichen Unterschiede in der Beitrittsbereitschaft zwischen Ost- und West wurden nach dem Gesamtmodell Modelle für Ost- wie Westdeutschland und Messzeitpunkt berechnet. So lassen sich mögliche Unterschiede in der Einflusskraft der Prädiktoren zwischen den Gebieten auffinden.

Die Hypothese 1a (hoher sozioökonomischer Status) wird durch die Analysen zu beiden Zeitpunkten nicht unterstützt; das betrifft auch Hypothese 1b (viel Zeit). Sowohl 2008 als auch 2018 sind die Haupteffekte des Nettoäquivalenzeinkommens, der zur Verfügung stehenden Zeit sowie der Bildung nicht signifikant. Damit zeigen die von uns untersuchten sozioökonomischen Variablen in den Berechnungen keinen Einfluss auf die Parteiaktivitätsbereitschaft. Allerdings scheint im Bereich der sozio-demografischen Faktoren das Alter einen Einfluss zu haben: Diese Variable ist signifikant in den Modellen enthalten. Demnach sinkt die Aktivitätsbereitschaft leicht mit steigendem Alter.

Die politische efficacy und das politische Interesse hingegen zeigen einen deutlich stärkeren Einfluss auf die Parteimitarbeitsbereitschaft. Damit werden die Hypothesen $2 \mathrm{a}$ und $2 \mathrm{~b}$ durch die Analyse gestützt. Die internal efficacy (das Selbstvertrauen, Politik zu verstehen), und eine Responsivitätswahrnehmung der Politik (external efficacy, Hypothese 2a) sowie das allgemeine politische Interesse (Hypothese 2b)

\footnotetext{
5 Dargestellt mithilfe von stargazer (Hlavac 2018).
} 
Tab. 2 Binomiale logistische Regressionsanalysen zur Erklärung der Parteiaktivitätsbereitschaft in Deutschland für 2008. (Eigene Berechnungen basierend auf dem ALLBUS 2008 nach Ausschluss aller Parteimitglieder, für das Gesamtmodell gewichtet anhand des personenbezogenen Ost-West-Gewichts)

\begin{tabular}{|c|c|c|c|}
\hline & \multicolumn{3}{|l|}{2008} \\
\hline & $\begin{array}{l}\text { Gesamt } \\
\text { (1) }\end{array}$ & $\begin{array}{l}\text { Ost } \\
(2)\end{array}$ & $\begin{array}{l}\text { West } \\
\text { (3) }\end{array}$ \\
\hline Geschlecht (Referenz: Frau) & $0,993(0,142)$ & $0,946(0,271)$ & $1,053(0,170)$ \\
\hline Alter & $\begin{array}{l}0,973 * * * \\
(0,005)\end{array}$ & $\begin{array}{l}0,966 * * * \\
(0,010)\end{array}$ & $\begin{array}{l}0,974 * * * \\
(0,006)\end{array}$ \\
\hline \multicolumn{4}{|l|}{ Bildung (Referenz: niedrig) } \\
\hline Mittel & $0,963(0,174)$ & $0,601(0,367)$ & $1,198(0,199)$ \\
\hline Hoch & $0,902(0,183)$ & $0,661(0,415)$ & $0,965(0,205)$ \\
\hline Familienstand (Referenz: verheiratet) & $1,214(0,142)$ & $1,113(0,290)$ & $1,345^{+}(0,167)$ \\
\hline Internal Efficacy & $1,256^{*}(0,098)$ & $1,219(0,189)$ & $1,266 *(0,116)$ \\
\hline External Efficacy & $\begin{array}{l}1,334 * * \\
(0,106)\end{array}$ & $1,219(0,223)$ & $\begin{array}{l}1,391 * * \\
(0,123)\end{array}$ \\
\hline Politisches Interesse & $\begin{array}{l}1,470 * * * \\
(0,088)\end{array}$ & $1,244(0,168)$ & $\begin{array}{l}1,596 * * * \\
(0,105)\end{array}$ \\
\hline Vertrauen in Parteien & $1,076(0,055)$ & $1,270 *(0,107)$ & $0,999(0,065)$ \\
\hline \multicolumn{4}{|l|}{ Berufstätigkeit (Referenz: Vollzeit) } \\
\hline Teilzeit & $1,229(0,190)$ & $2,281 *(0,374)$ & $0,968(0,214)$ \\
\hline Arbeitslos & $0,786(0,168)$ & $1,473(0,346)$ & $\begin{array}{l}0,577 * * \\
(0,201)\end{array}$ \\
\hline Vereinstätigkeit (Referenz: Tätig) & $0,996(0,153)$ & $1,466(0,312)$ & $0,858(0,178)$ \\
\hline $\begin{array}{l}\text { Soziale Eingebundenheit } \\
\text { (Zeit mit Freunden, Kollegen, etc.) }\end{array}$ & $1,051(0,102)$ & $1,122(0,212)$ & $1,053(0,118)$ \\
\hline Nettoäquivalenzeinkommen & $1,000(0,0001)$ & $1,000(0,0002)$ & $1,000(0,0001)$ \\
\hline Wohngebiet (Referenz: Ost) & $0,709 *(0,155)$ & - & - \\
\hline Konstante & $0,309^{+}(0,647)$ & $0,087^{+}(1,355)$ & $0,440(0,750)$ \\
\hline$N$ & 2461 & 838 & 1623 \\
\hline Log Likelihood & $-837,472$ & $-228,158$ & $-598,160$ \\
\hline$A I C$ & $1,706,945$ & 486,316 & $1,226,320$ \\
\hline McFadden & 0,287 & 0,248 & 0,309 \\
\hline
\end{tabular}

Odds Ratios und Standardfehler in Klammern

Die Spalte für Gesamtdeutschland nimmt Westdeutschland zur Referenz und Ostdeutschland als Koeffizient

Für das Geschlecht dient ,Mann“ als Referenz, beim Familienstand ,unverheiratet‘, bei Berufstätigkeit ,Vollzeit‘, bei Vereinstätigkeit ,nicht vereinstätig‘, für die Bildung; niedrige Bildung (maximal Hauptschulabschluss)

Die abhängige Variable ist dichotom codiert $(0=$ nicht beitrittswillig, $1=$ beitrittswillig $)$

${ }^{+} p<0,1 ; * p<0,05 ; * * p<0,01 ; * * * p<0,001$

erhöhen die Wahrscheinlichkeit signifikant, in einer politischen Partei mitarbeiten zu wollen. Menschen sind also stärker zu einer Aktivität in einer politischen Partei bereit, wenn sie davon ausgehen, dass ihre Gedanken Gehör finden und sie sich fähig dazu fühlen, in einer politischen Gruppe aktiv zu werden.

Allerdings ist anzumerken, dass sich die politische efficacy deutlich zwischen Ost und West unterscheidet: In Ostdeutschland verfügen alle Befragten laut den deskrip- 
Tab. 3 Binomiale logistisch Regressionsanalysen zur Erklärung der Parteiaktivitätsbereitschaft in Deutschland für 2018. (Eigene Berechnungen basierend auf dem ALLBUS 2018 nach Ausschluss aller Parteimitglieder, für das Gesamtmodell gewichtet anhand des personenbezogenen Ost-West-Gewichts)

\begin{tabular}{|c|c|c|c|}
\hline & \multicolumn{3}{|l|}{2018} \\
\hline & $\begin{array}{l}\text { Gesamt } \\
\text { (1) }\end{array}$ & $\begin{array}{l}\text { Ost } \\
(2)\end{array}$ & $\begin{array}{l}\text { West } \\
(3)\end{array}$ \\
\hline Geschlecht (Referenz: Frau) & $1,134(0,115)$ & $0,905(0,247)$ & $1,197(0,130)$ \\
\hline Alter & $\begin{array}{l}0,983 * * * \\
(0,004)\end{array}$ & $0,981 *(0,009)$ & $\begin{array}{l}0,984 * * * \\
(0,005)\end{array}$ \\
\hline \multicolumn{4}{|l|}{ Bildung (Referenz: niedrig) } \\
\hline Mittel & $0,948(0,160)$ & $0,974(0,416)$ & $0,947(0,176)$ \\
\hline Hoch & $1,142(0,165)$ & $1,387(0,448)$ & $1,106(0,179)$ \\
\hline Familienstand (Referenz: verheiratet) & $0,997(0,113)$ & $0,739(0,250)$ & $1,072(0,128)$ \\
\hline Internal Efficacy & $1,169^{+}(0,091)$ & $0,968(0,192)$ & $1,210^{+}(0,103)$ \\
\hline External Efficacy & $1,072(0,094)$ & $1,002(0,200)$ & $1,097(0,107)$ \\
\hline Politisches Interesse & $\begin{array}{l}1,509 * * * \\
(0,077)\end{array}$ & $1,504 *(0,165)$ & $\begin{array}{l}1,508 * * * \\
(0,087)\end{array}$ \\
\hline Vertrauen in Parteien & $\begin{array}{l}1,137 * * \\
(0,050)\end{array}$ & $1,297 *(0,108)$ & $1,102^{+}(0,056)$ \\
\hline \multicolumn{4}{|l|}{ Berufstätigkeit (Referenz: Vollzeit) } \\
\hline Teilzeit & $0,761^{+}(0,160)$ & $0,694(0,412)$ & $0,747^{+}(0,175)$ \\
\hline Arbeitslos & $0,858(0,147)$ & $0,935(0,315)$ & $0,833(0,167)$ \\
\hline Vereinstätigkeit (Referenz: Tätig) & $1,219(0,133)$ & $\begin{array}{l}2,350 * * \\
(0,286)\end{array}$ & $1,010(0,151)$ \\
\hline Nettoäquivalenzeinkommen & $1,000(0,0001)$ & $1,000(0,0001)$ & $1,000(0,0001)$ \\
\hline $\begin{array}{l}\text { Soziale Eingebundenheit } \\
\text { (Zeit mit Freunden, Kollegen, etc.) }\end{array}$ & $0,853^{+}(0,087)$ & $0,994(0,185)$ & $0,815^{*}(0,099)$ \\
\hline Wohngebiet (Referenz: Ost) & $\begin{array}{l}0,467 * * * \\
(0,132)\end{array}$ & - & - \\
\hline Konstante & $0,976(0,600)$ & $0,380(1,342)$ & $1,035(0,675)$ \\
\hline$N$ & 2667 & 870 & 1797 \\
\hline Log Likelihood & $-1,168,545$ & $-266,117$ & $-896,094$ \\
\hline$A I C$ & $2,369,090$ & 562,235 & $1,822,188$ \\
\hline McFadden & 0,244 & 0,220 & 0,233 \\
\hline
\end{tabular}

Odds Ratios und Standardfehler in Klammern

Die Spalte für Gesamtdeutschland nimmt Westdeutschland zur Referenz und Ostdeutschland als Koeffizient

Für das Geschlecht dient ,Mann“ als Referenz, beim Familienstand ,unverheiratet', bei Berufstätigkeit ,Vollzeit‘, bei Vereinstätigkeit ,nicht vereinstätig‘, für die Bildung; niedrige Bildung (maximal Hauptschulabschluss)

Die abhängige Variable ist dichotom codiert $(0=$ nicht beitrittswillig, $1=$ beitrittswillig)

${ }^{+} p<0,1 ; * p<0,05 ; * * p<0,01 ; * * * p<0,001$

tiven Analysen sowohl über eine geringere internal efficacy als auch eine geringere external efficacy. Für 2008 ergeben sich geringe, aber dennoch statistische signifikante Unterschiede $(p<0,05)$ zwischen West und Ost für die external efficacy (West: $\mathrm{M}=2,13 ; \mathrm{SD}=0,69$; Ost: $\mathrm{M}=1,92 ; \mathrm{SD}=0,65)$ sowie bei der internal efficacy (West: $\mathrm{M}=2,71 ; \mathrm{SD}=0,90 ;$ Ost: $\mathrm{M}=2,70 ; \mathrm{SD}=0,87)$. Ebensolche Werte und signifikanten Unterschiede $(p<0,05)$ zeigen sich auch für 2018 bei der external efficacy (West: 
$\mathrm{M}=2,28 ; \mathrm{SD}=0,67 ;$ Ost: $\mathrm{M}=2,11 ; \mathrm{SD}=0,69)$ und für die internal efficacy (West: $\mathrm{M}=2,86 ; \mathrm{SD}=0,80 ;$ Ost: $\mathrm{M}=2,77 ; \mathrm{SD}=0,81)$.

Das Vertrauen in politische Parteien hat dagegen zumindest in Ostdeutschland einen signifikanten Einfluss in den Modellen. Je höher das Vertrauen, desto wahrscheinlicher ist die individuelle Aktivitätsbereitschaft. Zudem bildet das politische Interesse zu beiden Messzeitpunkten eine relevante Einflussgröße. Insgesamt zeigen sich Unterschiede zwischen den beiden Zeitpunkten: Während die internal efficacy und politisches Interesse kontinuierlich wesentliche Faktoren darstellen, besitzt die external efficacy keinen signifikanten Einfluss auf die Parteimitarbeitsbereitschaft zum zweiten Untersuchungszeitpunkt (vgl. Tab. 2). Dies lässt sich möglicherweise so erklären, dass external efficacy auf zweierlei Weise wirken kann: Und zwar durch den Glauben, dass Parteien prinzipiell eine geeignete Beteiligungsform darstellen, die den Bürgerinnen und Bürgern Gehör verschaffen. Eine mangelnde Responsivität der politischen Eliten könnte ebenso motivieren, selbst aktiv zu werden (ggf. außerhalb etablierter Parteien). Insgesamt wird durch diese beiden Aspekte eine Parteimitarbeitsbereitschaft zu beiden Zeitpunkten gesteigert.

Hypothese 3 (soziale Netzwerke) lässt sich nicht stützen: Es finden sich keine entsprechenden Hinweise auf erhöhte Wahrscheinlichkeiten für die Zeit, die Personen mit anderen, also Bekannten, Freundinnen und Freunden oder Kolleginnen oder Kollegen verbringen. Ebenso spielt die Eingebundenheit in Vereine keine Rolle für die allgemeine Parteimitarbeitsbereitschaft. Nur 2018 in Ostdeutschland lässt sich hier ein Effekt nachweisen.

Hypothese 4 (Hohes Vertrauen in politische Parteien führt zu einer gesteigerten Parteimitarbeitsbereitschaft) kann in dieser Analyse klar gestützt werden. Hier besteht ein positiver Zusammenhang, dieser ist für 2018 und zu beiden Messzeitpunkten für Ostdeutschland signifikant.

\section{Diskussion und Schluss}

Passend zur bisherigen Forschung zeigt sich, dass ein höherer sozioökonomischer Status allein die Aktivitätsbereitschaft in einer Partei nicht zu beeinflussen scheint. So sind es sowohl eher die efficacy als auch das politische Interesse, die wesentliche Einflussfaktoren darstellen. Diese hängen - ob vermittelt oder unvermittelt - jedoch mit dem sozioökonomischen Status zusammen (Whiteley 2012; Rattinger 2009; Niedermayer 2005). Das Vertrauen in Parteien ist vor allem in Ostdeutschland eine relevante Größe und überschattet hier den Einfluss der external efficacy.

Auch der geringe Einfluss von Netzwerken überrascht. Angenommen wurde, dass Netzwerke im Sinne des CVM als Trigger dienen und partizipationsfördernd, ja gar auslösend wirken können. Hier ist jedoch die abhängige Variable selbst nicht dazu geeignet, tatsächliche Partizipation zu messen, beinhaltet sie letztlich doch nur eine Partizipationsabsicht. Insofern wäre hier eine Adaption der Messinstrumente sinnvoll, um künftig tatsächliche Partizipationsformen sowie die Einbindung in Netzwerke genauer zu erfassen. In dieser Studie wurde lediglich eine dichotome Variable zur Einbindung in Netzwerke und zur genutzten Zeit mit Mitmenschen 
genutzt. Eine detailliertere Analyse wäre wünschenswert, sofern die Umfragedaten es zulassen.

Äquivalent zu Studien zur Parteimitgliedschaft zeigt sich, dass das Alter der Befragten einen signifikanten Faktor für die Parteiaktivitätsbereitschaft darstellt. Dies ist insofern eine wertvolle Erkenntnis, als Alterseffekte auf politische Partizipation bisher nicht abschließend ermittelt werden konnten (Lamprianou 2013; Jennings und Markus 1988). Aus unserer Analyse kann man schließen, dass gerade Alterseffekte vor Effekten der Berufstätigkeit, des Einkommens und anderer Faktoren hervortreten. Das verweist auf jene Personen in der so genannten ,rush-hour des Lebens ', die zwar beitrittsbereit, aber für Parteien schwer erreichbar sind, weil sie mit Berufstätigkeit und Familie voll ausgelastet sind.

Mit der Feststellung von Achury et al. (2018), dass Unterstützerinnen und Unterstützer von Parteien den Mitgliedern andere Beitrittsmotive zuschreiben als diejenigen Gründe, die tatsächlich zum Beitritt bei Parteimitgliedern geführt haben, kann ein Teil der Erklärungslücke hinsichtlich der Forschungsfrage dieses Beitrags geschlossen werden. Wir zeigen, dass es vor allem das politische Interesse sowie das Vertrauen in Parteien ist, das Menschen dazu bewegt, in Parteien mitzuarbeiten. Insofern trägt also vor allem intrinsische Motivation dazu bei, Menschen in die Parteien zu bringen. Parteimitarbeitsbereite - sowie bei Poletti et al. (2018) aktive Unterstützerinnen und Unterstützerinnen Unterstützer - scheinen eine Vorstellung davon zu haben, was Parteimitglieder zu ihrem Beitritt bewegt hat. „[P]arty supporters believe that party members must derive intrinsic pleasure from involvement in party life, and career or material benefits, and that they must be embedded in a social network that draws them into the party [...]." (Poletti et al. 2018, S. 12f.). Gerade die Vermutung des positiven Einflusses einer umfassenden Einbettung in soziale Netzwerke können wir in dieser Studie nicht bestätigen, was auch an der Messung der unabhängigen Variablen liegen kann.

Die vorliegenden Ergebnisse des politischen Interesses und Vertrauens bestätigen die Analyse von Achury et al. (2018) dahingehend, dass Parteien mit ideellen Angeboten eher Zulauf erhalten können und kongruentere Mitgliedsbasen entwickeln als Parteien, deren Anreizstrukturen in erster Linie auf Kosten-Nutzen-Kalkülen basieren. Einen Zusammenhang zwischen sozio-demografischen Faktoren oder der Einbindung in Netzwerke der jeweiligen Parteimitglieder zeigt auch die Studie von Achury et al. (2018) nicht, wohingegen die vorliegenden Ergebnisse in die Richtung eines Zusammenhangs dieser Faktoren deuten. Als weitere Limitation der Untersuchung ist anzuführen, dass bei der Frage nach einer Beitrittsbereitschaft in Parteien in Umfragen nicht ausgeschlossen werden kann, dass Effekte der wahrgenommenen sozialen Erwünschtheit zum Tragen kommen.

Dieser Beitrag untersuchte die Determinanten im Hinblick auf Wahrscheinlichkeiten zur Parteiaktivität bzw. zur Parteibeitrittsbereitschaft. Die Modelle weisen eindeutig darauf hin, dass es weiterer Untersuchungen bedarf, die das CVM erweitern (etwa um Netzwerke bzw. andere Operationalisierungen von Netzwerken) und im Rahmen explorativer Feldzugänge (wie ethnografische Mikrostudien) die tieferliegenden Ursachen einer gleichzeitigen Parteiaktivitätsbereitschaft bei sinkenden Eintrittszahlen analysieren. Die Studie von Poletti et al. (2018) etwa verweist darauf, dass es sich hierbei um ein Phänomen kollektiver policy-Anreize handeln 
könnte (8), weil sich die Motive der Parteibeitrittsbereiten von den Erwartungen der bereits organisierten Parteimitglieder unterscheiden und insofern ein Mismatch zwischen Perzeption und Realität vorliegt.

Wie also diejenigen Bürgerinnen und Bürger, die sich eine Parteiaktivität vorstellen können, ,ticken“, konnte im Rahmen dieses Beitrages exploriert werden. Daher schließt der Beitrag mit einem Plädoyer an die Forschung, sich dem Thema weiter anzunehmen - das überraschend große Aktivitätspotenzial vor allem unter Jugendlichen (Gaiser und de Rijke 2016) sollte auch von der Parteienforschung stärker bearbeitet werden. Denn gerade in dieser Zielgruppe scheint Potenzial für Parteien zu liegen, wie ja neue Bewegungen wie Fridays for Future zeigen. Nicht zuletzt die Aktivitäten der Bewegung zeigen, dass Jugendliche politisch engagiert sind und aktiv agieren. Inwiefern aus dieser sozialen Bewegung Parteigründungen hervorgehen oder bestehende Parteien neue Wählerinnen und Wähler binden können, sollte durch zukünftige Forschung ermittelt werden. Zudem zeigen Parteigründungen wie die von Volt und der AfD mit ihren Verbindungen zur Identitären Bewegung (Boehnke 2019), dass sich Parteien aktiv den Jüngeren zuwenden und es womöglich hier einer differenzierenden Analyse bedarf. Dies gilt auch für Entwicklungen zu weiteren gesellschaftlich kontroversen Themen entlang neuer Cleavages wie Migration, Klimafragen oder Europa, über die Personen zur Mitarbeit in Parteien bewegt werden können.

\section{Anhang}

Tab. 4 Deskriptive Statistiken für Parteimitarbeitsbereite und Parteimitglieder in 2008. (Quelle: Eigene Berechnungen)

\begin{tabular}{|c|c|c|c|c|c|c|c|}
\hline Variable & $\begin{array}{l}\text { Nicht-Partei- } \\
\text { mitarbeitsberei- } \\
\text { te (Mittelwert) }\end{array}$ & $N$ & $\begin{array}{l}\text { Parteimitarbeits- } \\
\text { bereite } \\
\text { (Mittelwert) }\end{array}$ & $N$ & $\begin{array}{l}\text { Parteimit- } \\
\text { glieder } \\
\text { (Mittel- } \\
\text { wert) }\end{array}$ & $N$ & Sig \\
\hline Geschlecht & 1,52 & 2841 & 1,46 & 383 & 1,30 & 101 & $*$ \\
\hline Mann & 48,46 & 1377 & 53,79 & 206 & 70,29 & 71 & - \\
\hline Frau & 51,53 & 1464 & 46,21 & 177 & 29,70 & 30 & - \\
\hline Alter & 51,04 & 2829 & 43,27 & 383 & 54,05 & 101 & $* * *$ \\
\hline Familienstand & 0,58 & 2838 & 0,57 & 382 & 0,69 & 101 & ns \\
\hline Nicht verheiratet & 40,69 & 1155 & 42,67 & 163 & 30,69 & 31 & - \\
\hline $\begin{array}{l}\text { Verheiratet/ } \\
\text { zusammenlebend }\end{array}$ & 57,97 & 1645 & 57,33 & 219 & 69,31 & 70 & - \\
\hline $\begin{array}{l}\text { Nettoäquivalenz- } \\
\text { einkommen }\end{array}$ & 1462,81 & 2325 & 1666,96 & 307 & 2005,65 & 86 & $* * *$ \\
\hline Schulabschluss & 1,87 & 2797 & 2,13 & 371 & 2,13 & 101 & $\mathrm{~ns}$ \\
\hline Niedrig & 40,10 & 1137 & 2,15 & 8 & 31,68 & 32 & - \\
\hline Mittel & 31,70 & 898 & 57,68 & 214 & 23,76 & 24 & - \\
\hline Hoch & 26,90 & 762 & 40,16 & 149 & 44,55 & 45 & - \\
\hline Berufstätigkeit & 2,09 & 2841 & 1,86 & 383 & 1,92 & 101 & $* * *$ \\
\hline Vollzeit & 39,0 & 1107 & 49,35 & 189 & 49,50 & 50 & - \\
\hline Teilzeit & 12,6 & 360 & 18,02 & 69 & 8,91 & 9 & - \\
\hline
\end{tabular}


Tab. 4 (Fortsetzung)

\begin{tabular}{|c|c|c|c|c|c|c|c|}
\hline Variable & $\begin{array}{l}\text { Nicht-Partei- } \\
\text { mitarbeitsberei- } \\
\text { te (Mittelwert) }\end{array}$ & $N$ & $\begin{array}{l}\text { Parteimitarbeits- } \\
\text { bereite } \\
\text { (Mittelwert) }\end{array}$ & $N$ & $\begin{array}{l}\text { Parteimit- } \\
\text { glieder } \\
\text { (Mittel- } \\
\text { wert) }\end{array}$ & $N$ & Sig \\
\hline Arbeitslos & 48,4 & 1374 & 32,64 & 125 & 41,58 & 42 & - \\
\hline $\begin{array}{l}\text { Treiman-Presti- } \\
\text { geskala (I68) }\end{array}$ & 42,48 & 1308 & 3,99 & 231 & 4,37 & 38 & $* * *$ \\
\hline Internal Efficacy & 2,67 & 2803 & 3,01 & 382 & 3,43 & 101 & $* * *$ \\
\hline $\begin{array}{l}\text { External Effica- } \\
\text { cy }\end{array}$ & 2,03 & 2752 & 2,25 & 381 & 2,45 & 101 & $* * *$ \\
\hline $\begin{array}{l}\text { Politisches Inte- } \\
\text { resse }\end{array}$ & 2,99 & 2839 & 2,56 & 383 & 1,76 & 101 & $* * *$ \\
\hline $\begin{array}{l}\text { Vertrauen in po- } \\
\text { litische Parteien }\end{array}$ & 3,06 & 2767 & 3,41 & 381 & 3,57 & 101 & $* * *$ \\
\hline $\begin{array}{l}\text { Mitgliedschaft } \\
\text { in Vereinen }\end{array}$ & 0,51 & 2833 & 0,60 & 381 & 0,83 & 101 & $* * *$ \\
\hline $\begin{array}{l}\text { Nicht vereinstä- } \\
\text { tig }\end{array}$ & 48,74 & 1381 & 39,90 & 152 & 16,83 & 17 & - \\
\hline Vereinstätig & 51,25 & 1452 & 60,10 & 229 & 83,16 & 84 & - \\
\hline $\begin{array}{l}\text { Zeitaufwand für } \\
\text { Kollegen und } \\
\text { Freunde }\end{array}$ & 3,46 & 2839 & 3,22 & 383 & 3,03 & 101 & $* * *$ \\
\hline Erhebungsgebiet & 0,32 & 2841 & 0,23 & 383 & 0,19 & 101 & ns \\
\hline Ost & 32,00 & 919 & 23,50 & 90 & 18,81 & 19 & - \\
\hline West & 67,00 & 1922 & 76,50 & 293 & 81,19 & 82 & - \\
\hline
\end{tabular}

Die Ergebnisse basieren auf ungewichteten Berechnungen anhand der ALLBUS-Daten 2008

Die Zellen zeigen Mittelwerte für metrische Variablen und prozentuale Anteile für kategorielle Variablen, die Anzahl der Befragten, sowie die Signifikanzniveaus von ANOVA-Mittelwertvergleichen für die metrischen Variablen und Chi-Quadrat-Tests für die kategoriellen Variablen zwischen den drei Gruppen $* p<0,05 ; * * p<0,01 ; * * * p<0,001$

Tab. 5 Deskriptive Statistiken für Parteimitarbeitsbereite und Parteimitglieder in 2018. (Quelle: Eigene Berechnungen)

\begin{tabular}{|c|c|c|c|c|c|c|c|}
\hline Variable & $\begin{array}{l}\text { Nicht-Partei- } \\
\text { mitarbeitsberei- } \\
\text { te (Mittelwert) }\end{array}$ & $N$ & $\begin{array}{l}\text { Parteimitarbeits- } \\
\text { bereite } \\
\text { (Mittelwert) }\end{array}$ & $N$ & $\begin{array}{l}\text { Parteimit- } \\
\text { glieder } \\
\text { (Mittel- } \\
\text { wert) }\end{array}$ & $N$ & Sig \\
\hline Geschlecht & 1,51 & 2705 & 1,45 & 588 & 1,35 & 121 & ns \\
\hline Mann & 49,43 & 1337 & 55,27 & 325 & 65,29 & 79 & - \\
\hline Frau & 49,54 & 1368 & 44,73 & 263 & 34,71 & 42 & - \\
\hline Alter & 50,57 & 2700 & 46,73 & 588 & 56,13 & 121 & $* * *$ \\
\hline Familienstand & 0,56 & 2699 & 2,73 & 585 & 0,63 & 121 & ns \\
\hline Nicht verheiratet & 43,83 & 1183 & 48,72 & 285 & 37,19 & 45 & - \\
\hline $\begin{array}{l}\text { Verheiratet/ } \\
\text { zusammenlebend }\end{array}$ & 56,17 & 1516 & 51,28 & 300 & 62,81 & 76 & - \\
\hline $\begin{array}{l}\text { Nettoäquivalenz- } \\
\text { einkommen }\end{array}$ & 1917,35 & 2374 & 2092,91 & 534 & 2453,32 & 107 & $* * *$ \\
\hline Schulabschluss & 2,10 & 2676 & 2,36 & 574 & 2,40 & 121 & ns \\
\hline
\end{tabular}


Tab. 5 (Fortsetzung)

\begin{tabular}{|c|c|c|c|c|c|c|c|}
\hline Variable & $\begin{array}{l}\text { Nicht-Partei- } \\
\text { mitarbeitsberei- } \\
\text { te (Mittelwert) }\end{array}$ & $N$ & $\begin{array}{l}\text { Parteimitarbeits- } \\
\text { bereite } \\
\text { (Mittelwert) }\end{array}$ & $N$ & $\begin{array}{l}\text { Parteimit- } \\
\text { glieder } \\
\text { (Mittel- } \\
\text { wert) }\end{array}$ & $N$ & Sig \\
\hline Niedrig & 26,30 & 704 & 1,16 & 8 & 19,01 & 23 & - \\
\hline Mittel & 36,95 & 989 & 34,88 & 253 & 22,31 & 27 & - \\
\hline Hoch & 36,73 & 983 & 63,95 & 313 & 58,68 & 71 & - \\
\hline Berufstätigkeit & 1,96 & 2705 & 1,74 & 588 & 1,98 & 121 & $* *$ \\
\hline Vollzeit & 43,51 & 1177 & 54,93 & 323 & 41,32 & 50 & - \\
\hline Teilzeit & 16,49 & 446 & 15,82 & 93 & 19,01 & 23 & - \\
\hline Arbeitslos & 40,0 & 1082 & 29,25 & 172 & 39,67 & 48 & - \\
\hline $\begin{array}{l}\text { Treiman-Presti- } \\
\text { geskala (SIOPS) }\end{array}$ & 32,92 & 1942 & 48,25 & 421 & 50,87 & 69 & $* * *$ \\
\hline Internal Efficacy & 2,77 & 2680 & 3,06 & 585 & 3,46 & 121 & $* * *$ \\
\hline $\begin{array}{l}\text { External Effica- } \\
\text { cy }\end{array}$ & 2,18 & 2628 & 2,34 & 576 & 2,64 & 120 & *** $*$ \\
\hline $\begin{array}{l}\text { Politisches Inte- } \\
\text { resse }\end{array}$ & 2,78 & 2704 & 2,40 & 588 & 1,77 & 121 & $* * *$ \\
\hline $\begin{array}{l}\text { Vertrauen in po- } \\
\text { litische Parteien }\end{array}$ & 3,39 & 2637 & 3,70 & 581 & 4,08 & 120 & $* * *$ \\
\hline $\begin{array}{l}\text { Mitgliedschaft } \\
\text { in Vereinen }\end{array}$ & 0,54 & 2703 & 0,69 & 587 & 0,85 & 121 & $* * *$ \\
\hline $\begin{array}{l}\text { Nicht vereinstä- } \\
\text { tig }\end{array}$ & 45,62 & 1233 & 30,83 & 181 & 14,87 & 18 & - \\
\hline Vereinstätig & 54,38 & 1470 & 69,17 & 406 & 85,12 & 103 & - \\
\hline $\begin{array}{l}\text { Zeitaufwand für } \\
\text { Kollegen und } \\
\text { Freunde }\end{array}$ & 3,36 & 2705 & 3,10 & 574 & 3,02 & 118 & $* * *$ \\
\hline Erhebungsgebiet & 0,35 & 2705 & 1,18 & 588 & 0,15 & 121 & ns \\
\hline Ost & 34,89 & 944 & 17,86 & 105 & 14,87 & 18 & - \\
\hline West & 65,10 & 1761 & 82,14 & 483 & 85,12 & 103 & - \\
\hline
\end{tabular}

Die Ergebnisse basieren auf ungewichteten Berechnungen anhand der ALLBUS-Daten 2018

Mittelwerte für metrische Variablen und prozentuale Anteile für kategorielle Variablen, die Anzahl der Befragten, sowie die Signifikanzniveaus von ANOVA-Mittelwertvergleichen für die metrischen Variablen und Chi-Quadrat-Tests für die kategoriellen Variablen zwischen den drei Gruppen

$* p<0,05 ; * * p<0,01 ; * * * p<0,001$ 


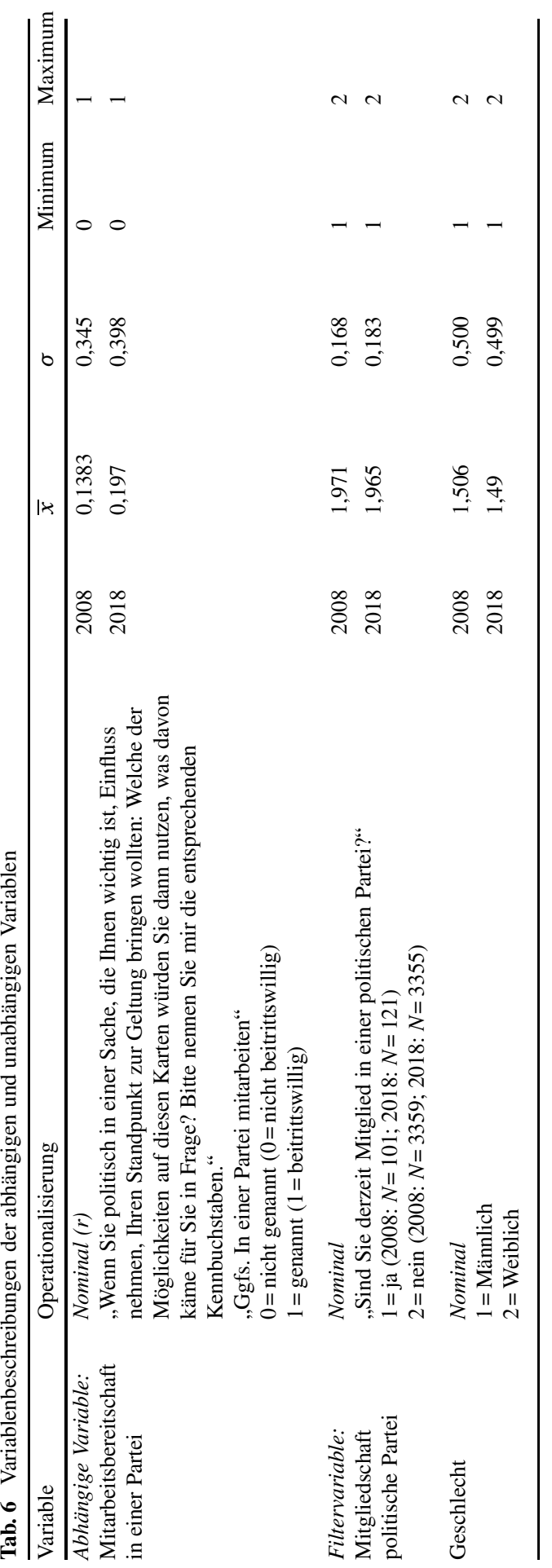




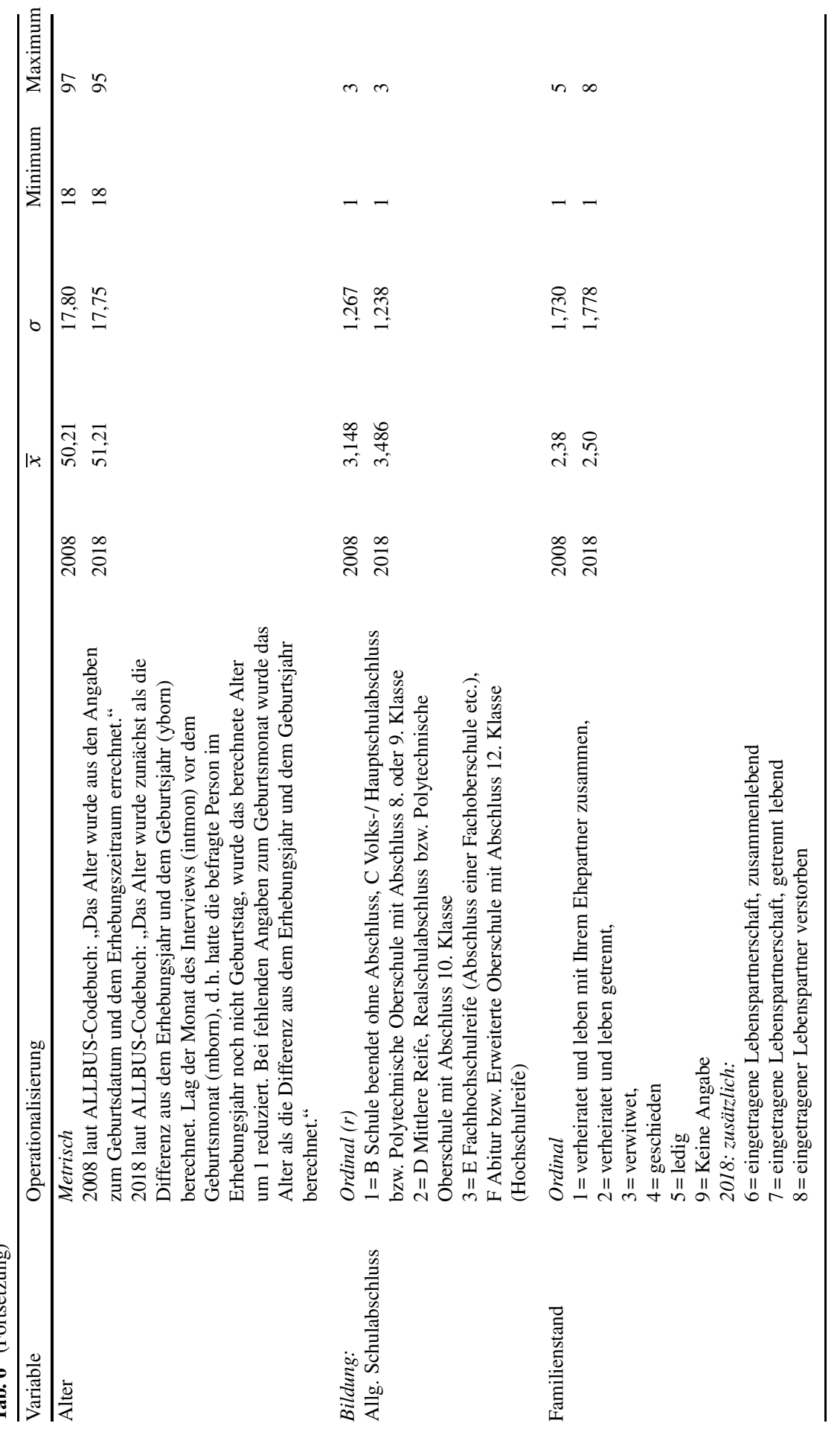




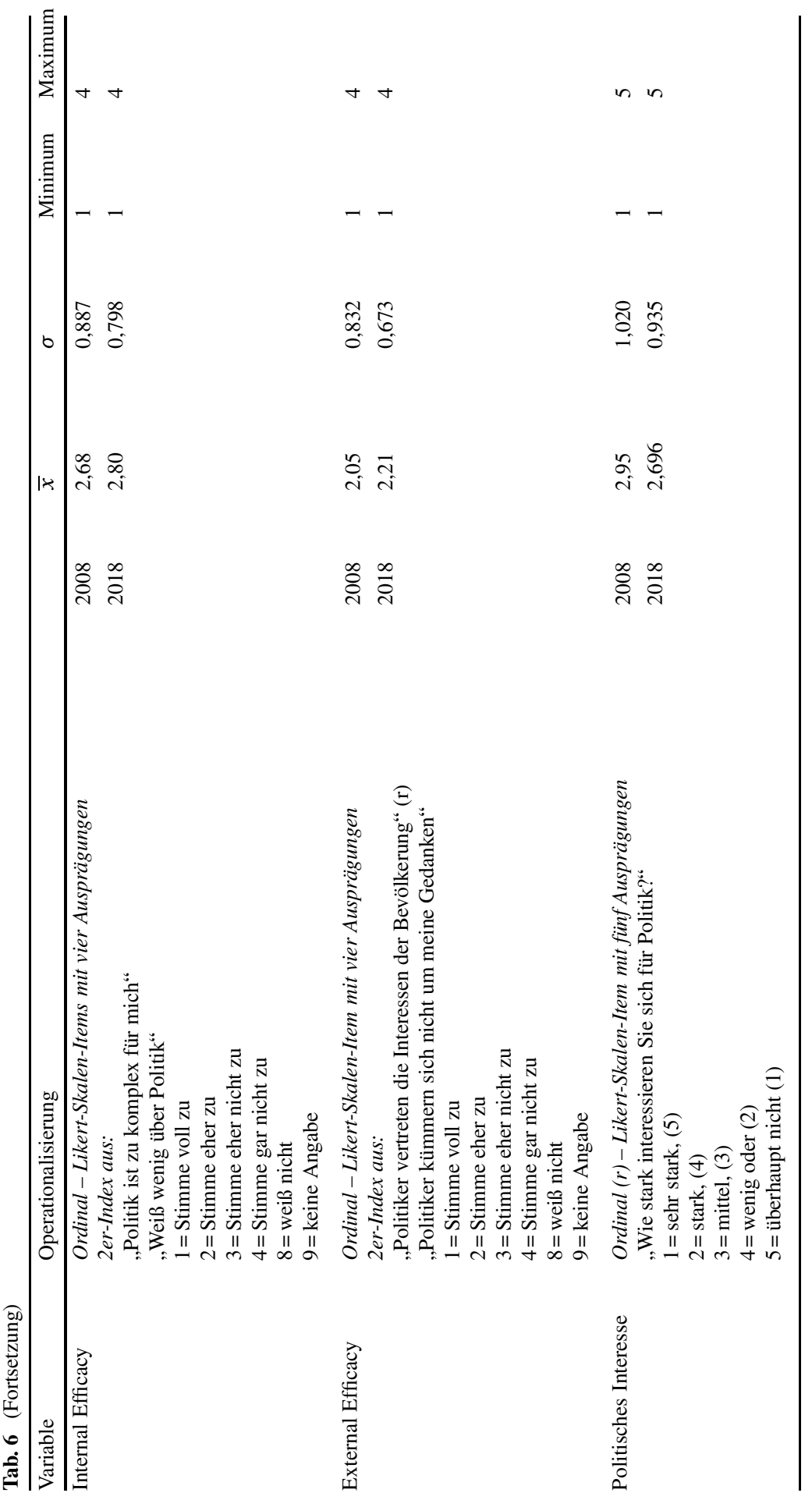




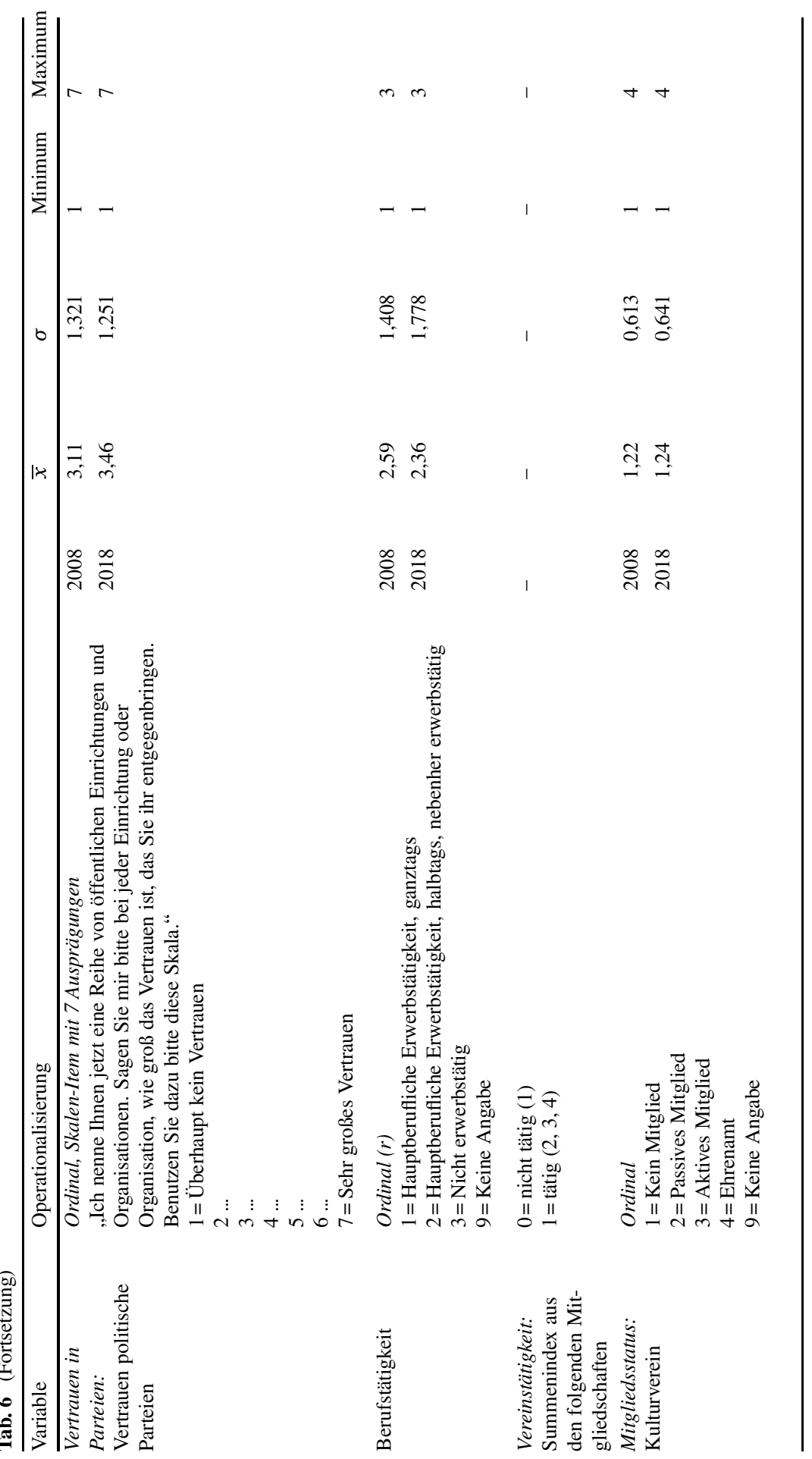




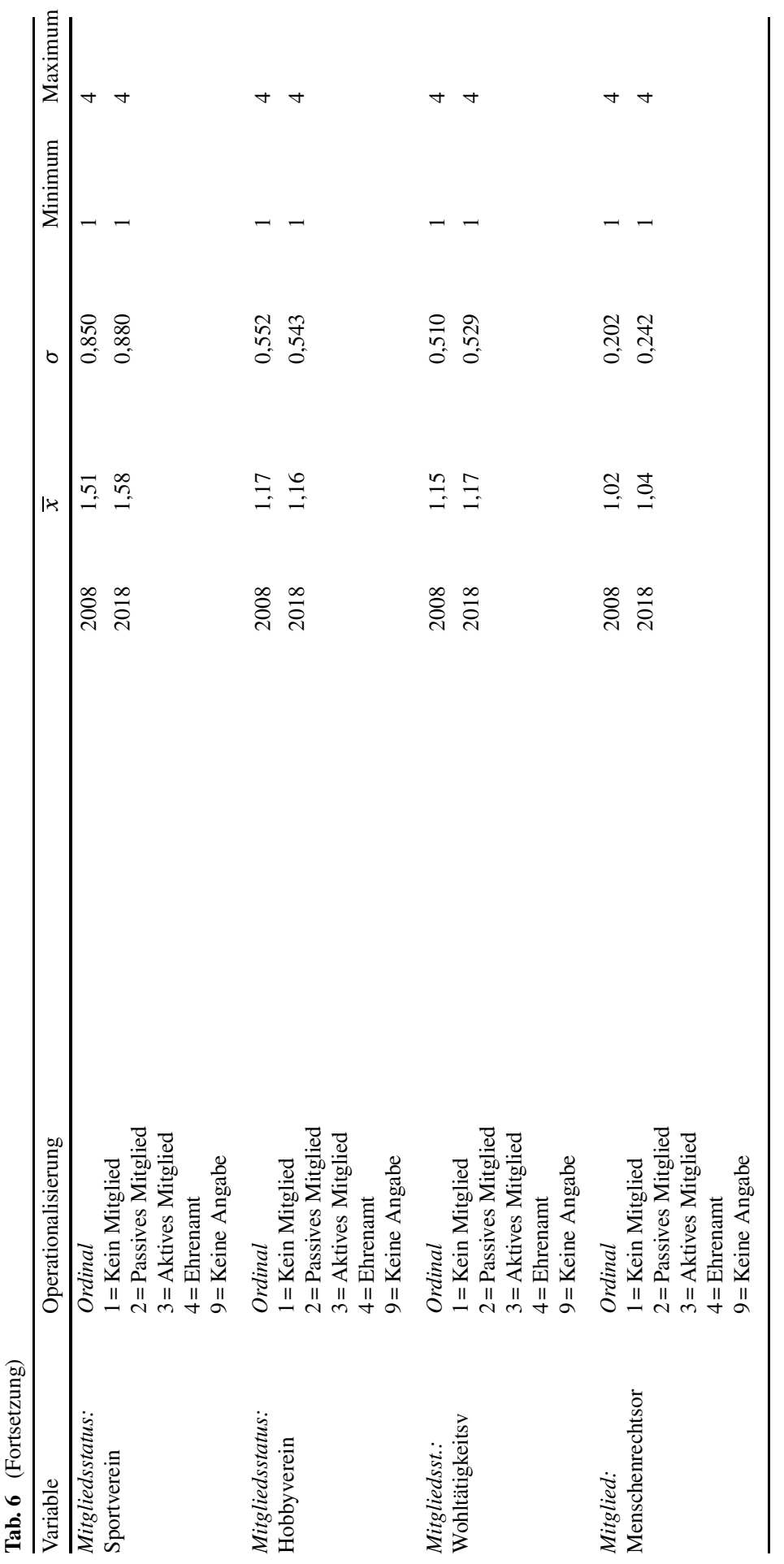




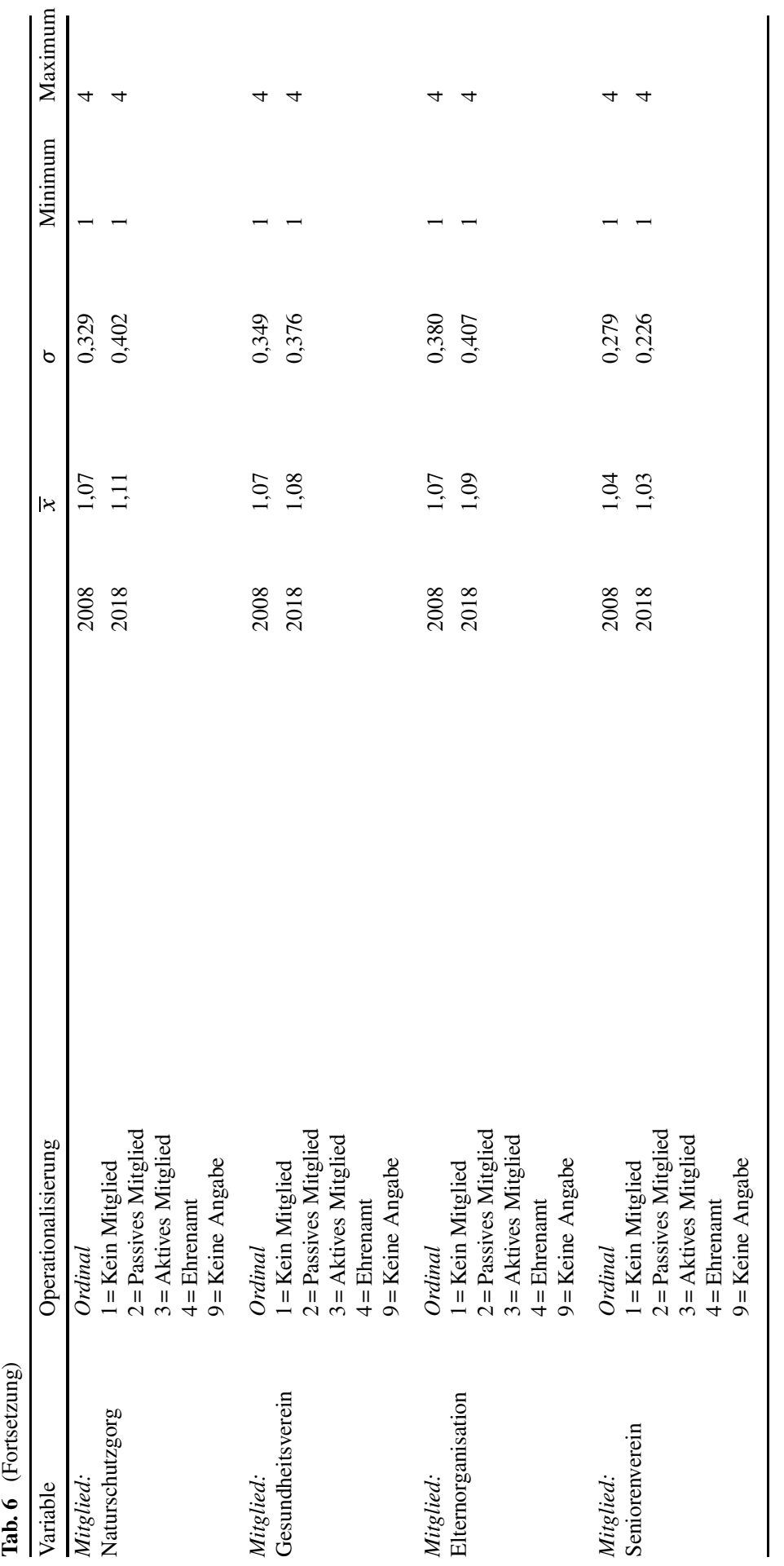




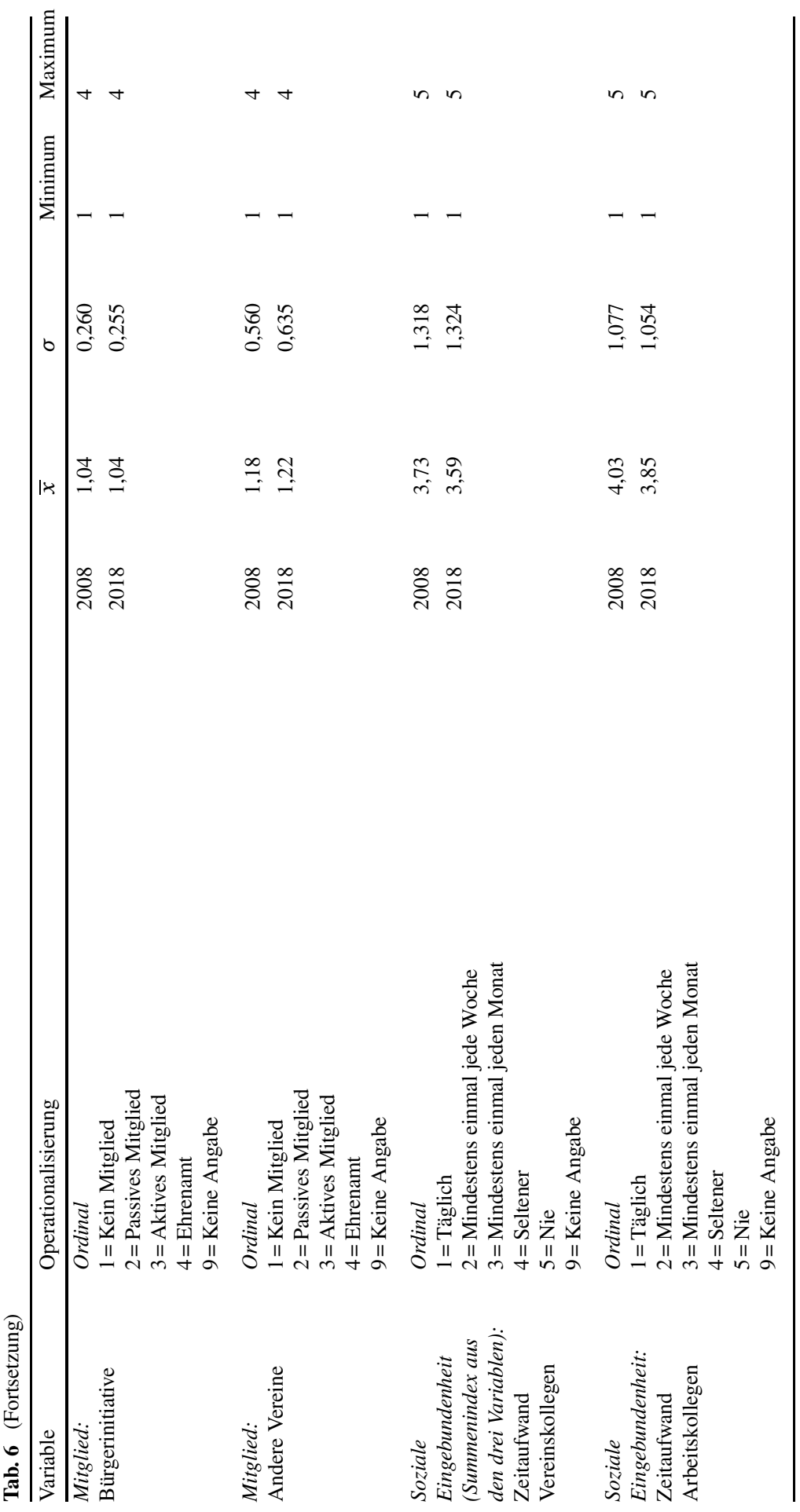




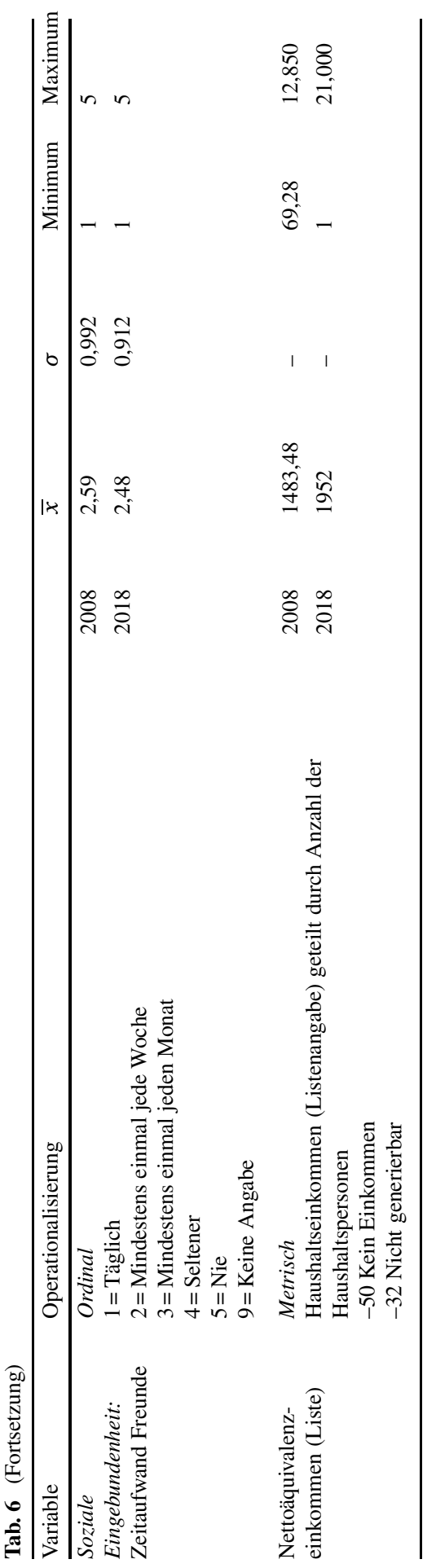


Funding Die Arbeit der Nachwuchsforschungsgruppe DIPART (geleitet von Isabelle Borucki) wird durch das Forschungsprogramm ,Digitale Gesellschaft“ des Ministeriums für Kultur und Wissenschaft des Landes Nordrhein-Westfalen finanziert (Fördernummer 005-1709-0003).

Funding Open Access funding enabled and organized by Projekt DEAL.

Open Access Dieser Artikel wird unter der Creative Commons Namensnennung 4.0 International Lizenz veröffentlicht, welche die Nutzung, Vervielfältigung, Bearbeitung, Verbreitung und Wiedergabe in jeglichem Medium und Format erlaubt, sofern Sie den/die ursprünglichen Autor(en) und die Quelle ordnungsgemäß nennen, einen Link zur Creative Commons Lizenz beifügen und angeben, ob Änderungen vorgenommen wurden.

Die in diesem Artikel enthaltenen Bilder und sonstiges Drittmaterial unterliegen ebenfalls der genannten Creative Commons Lizenz, sofern sich aus der Abbildungslegende nichts anderes ergibt. Sofern das betreffende Material nicht unter der genannten Creative Commons Lizenz steht und die betreffende Handlung nicht nach gesetzlichen Vorschriften erlaubt ist, ist für die oben aufgeführten Weiterverwendungen des Materials die Einwilligung des jeweiligen Rechteinhabers einzuholen.

Weitere Details zur Lizenz entnehmen Sie bitte der Lizenzinformation auf http://creativecommons.org/ licenses/by/4.0/deed.de.

\section{Literatur}

Achury, Susan, Susan E. Scarrow, Karina Kosiara-Pedersen, und Emilie van Haute. 2018. The consequences of membership incentives: Do greater political benefits attract different kinds of members? Party Politics https://doi.org/10.1177/1354068818754603.

Acik, Necla. 2013. Reducing the participation gap in civic engagement: political consumerism in Europe. European Sociological Review 29(6):1309-1322.

Almond, Gabriel, und Sidney Verba. 1989. The civic culture: political attitudes and democracy in five nations. Newbury Park: SAGE. 1. Auflage: 1963.

Arzheimer, Kai. 2016. Wahlverhalten in Ost-West-Perspektive. In Wahlen und Wähler, Analysen aus Anlass der Bundestagswahl 2013, Hrsg. Harald Schoen, Bernhard Weßels, 71-89. Wiesbaden: Springer.

Arzheimer, Kai, und Jürgen W. Falter. 2013. Versöhnen statt spalten? Das Ergebnis der Bundestagswahl 2009 und die Rolle der PDS/Linkspartei in Ost-West-Perspektive. In Wahlen und Wähler, Analysen aus Anlass der Bundestagswahl 2009, Hrsg. Bernhard Weßels, Harald Schoen, und Oscar W. Gabriel, 118-150. Wiesbaden: Springer.

Bardi, Luciano, Stefano Bartolini, und Alexander Trechsel. 2014. Party adaptation and change and the crisis of democracy. Party Politics 20(2):151-159.

Barnes, Samuel H., Klaus R. Allerbeck, Barbara G. Farah, Felix J. Heunks, Ronald F. Inglehart, M. Kent Jennings, Hans Dieter Klingemann, Alan Marsh, und Leopold Rosenmayr. 1979. Political action. Mass participation in five Western democracies. Beverly Hills: Sage.

Biehl, Heiko. 2013. Noch vertrauenswürdig? Konzept und Empirie des gesellschaftlichen Vertrauens in politische Parteien. In Abkehr von den Parteien? Parteiendemokratie und Bürgerprotest, Hrsg. Oskar Niedermayer, Benjamin Höhne, und Uwe Jun, 67-92. Wiesbaden: Springer.

Biezen, Ingrid van, Peter Mair, und Thomas Poguntke. 2012. Going, going...gone? The decline of party membership in contemporary Europe. European Journal of Political Research 51(1):24-56.

Boehnke, Lukas. 2019. Rechter Kulturkampf heute: Identitätskonstruktion und Framing-Strategien der Identitären Bewegung. In Rechtspopulismus im Fokus Theoretische und praktische Herausforderungen für die politische Bildung, Hrsg. Lukas Boehnke, Malte Thran, und Jacob Wunderwald, 89-114. Wiesbaden: Springer.

Bukow, Sebastian. 2013. Die professionalisierte Mitgliederpartei. Politische Parteien zwischen institutionellen Erwartungen und organisationaler Wirklichkeit. Wiesbaden: Springer.

Dahlgren, Peter. 2009. Media and political engagement: citizens, communication, and democracy. Communication, society and politics. Cambridge: Cambridge University Press.

Decker, Frank. 2015. Parteiendemokratie im Wandel. Beiträge zur Theorie und Empirie. Baden-Baden: Nomos. 
Deschouwer, Kris. 1996. Political parties and democracy: a mutual murder? European Journal of Political Research 29(3):263-278.

Dose, Nicolai, und Anne-Kathrin Fischer. 2013. Mitgliederschwund und Überalterung der Parteien: Prognose der Mitgliederzahlen bis 2040. ZParl 44(4):892-900.

Ebbinghaus, Bernhard, und Claudia Göbel. 2014. Mitgliederrückgang und Organisationsstrategien deutscher Gewerkschaften. In Handbuch Gewerkschaften in Deutschland, 2. Aufl., Hrsg. Wolfgang Schroeder, 207-240. Wiesbaden: Springer.

Eith, Ulrich, und Gerd Mielke. 2017. Gesellschaftlicher Strukturwandel und soziale Verankerung der Parteien. In Parteien und soziale Ungleichheit, 39-61. Wiesbaden: Springer.

Fabre, Elodie. 2008. Party organization in a multi-level system: party organizational change in Spain and the UK. Regional \& Federal Studies 18(4):309-329.

Falter, Jürgen W., und Harald Schoen. 2014. Glossar. In Handbuch Wahlforschung, 2. Aufl., Hrsg. Jürgen W. Falter, Harald Schoen, 869-884. Wiesbaden: Springer.

Fiorina, Morris P. 1999. Extreme voice: a dark side of civic engagement. In Civic engagement in American democracy, Hrsg. Theda Skocpol, Morris P. Fiorina, 395-425. Washington: Brookings Institution Press.

Gabriel, Oscar W. 2012. Political participation in France and Germany-traditions, concepts, measurements, patterns and explanations. In Political participation in France and Germany, Hrsg. Oscar W. Gabriel, Silke I. Keil, und Eric Kerrouche, 1-32. Colchester: ECPR Press.

Gabriel, Oscar W. 2018. Participation and representation: background and beliefs of activists and the inactive. In Political Representation in France and Germany, Hrsg. Oscar W. Gabriel, Eric Kerrouche, und Suzanne S. Schüttemeyer, 247-277. Cham: Springer.

Gaiser, Wolfgang, und Johann de Rijke. 2016. Jugend und politische Partizipation heute. In Jung, politisch, aktiv?! Politische Einstellungen und politisches Engagement junger Menschen. Ergebnisse der FESJugendstudie 2015, Hrsg. Wolfgang Gaiser, Stefanie Hanke, und Kerstin Ott, 50-71. Bonn: in.

Gauja, Anika. 2017. Party reform: the causes, challenges, and consequences of organizational change. Oxford: Oxford University Press.

Geißler, Rainer. 2014. Die Sozialstruktur Deutschlands, 7. Aufl., Wiesbaden: Springer VS.

Gerl, Katharina, Stefan Marschall, und Nadja Wilker. 2016. Innerparteiliche Demokratie 2.0? In Web 2.0 Demokratie 3.0. Digitale Medien und ihre Implikationen für Prozesse und Qualität von Demokratie Sonderheft der Zeitschrift für Vergleichende Politikwissenschaft., Hrsg. Marianne Kneuer, Samuel Salzborn, 115-149. Wiesbaden: Springer.

GESIS - Leibniz-Institut für Sozialwissenschaften. 2015. Allgemeine Bevölkerungsumfrage der Sozialwissenschaften ALLBUS 2008. GESIS Datenarchiv, Köln, ZA4600 v2.1.0, https://doi.org/10.4232/ 1.12345

GESIS - Leibniz-Institut für Sozialwissenschaften. 2019. Allgemeine Bevölkerungsumfrage der Sozialwissenschaften ALLBUS 2018. GESIS Datenarchiv, Köln, ZA5270 v2.0.0, https://doi.org/10.4232/ 1.13250 .

Gibson, Rachel, Fabienne Greffet, und Marta Cantijoch. 2017. Friend or foe? Political Communication 34:89-111.

Grunden, Timo, Maximilian Janetzki, und Julian Salandi. 2017. Die SPD. Anamnese einer Partei. BadenBaden: Nomos

Harfst, Philipp, Ina Kubbe, und Thomas Poguntke. 2017. Parties, governments and elites: the comparative study of democracy. Wiesbaden: Springer.

Harmel, Robert, und Kenneth Janda. 1994. An integrated theory of party goals and party change. Journal of Theoretical Politics 6(3):259-287.

Hlavac, Marek. 2018. stargazer: well-formatted regression and summary statistics tables. R package version 5.2.2. https://CRAN.R-project.org/package=stargazer. Zugegriffen: 5. Apr. 2020.

Holtmann, Everhard. 2012. Der Parteienstaat in Deutschland. Erklärungen, Entwicklungen, Erscheinungsbilder. Bonn: Bundeszentrale für politische Bildung.

Holtmann, Everhard. 2020a. Zusammenfassung der Ergebnisse. In Die Umdeutung der Demokratie. Politische Partizipation in Ost- und Westdeutschland, Hrsg. Everhard Holtmann, 323-342. Bonn: Bundeszentrale für politische Bildung.

Holtmann, Everhard (Hrsg.). 2020b. Die Umdeutung der Demokratie. Politische Partizipation in Ost- und Westdeutschland. Bonn: Bundeszentrale für politische Bildung.

Hooghe, Marc. 2014. Defining political participation: How to pinpoint an elusive target? Acta Politica 49(7):338-341.

Jaeck, Tobias. 2020. Die ,langen Wellen“ im Entwicklungsverlauf von politischer Partizipation in Ostdeutschland von 1990 bis zur Gegenwart. In Die Umdeutung der Demokratie. Politische Partizipation 
in Ost- und Westdeutschland, Hrsg. Everhard Holtmann, 35-56. Bonn: Bundeszentrale für politische Bildung.

Jakobs, Simon. 2021. Die Mitgliederwerbung von SPD und CDU im Vergleich. Strategielose Mitgliederpartei oder überfordertes Ehrenamt? Opladen: Budrich.

Jennings, M. Kent, und Gregory B. Markus. 1988. Political involvement in the later years, a longitudinal survey. American Journal of Political Science 32(2):302-316.

Jun, Uwe. 2009a. Politische Parteien als Gegenstand der Politischen Soziologie. In Politische Soziologie. Ein Studienbuch, Hrsg. Viktoria Kaina, Andrea Römmele, 235-266. Wiesbaden: Springer.

Jun, Uwe. 2009b. Organisationsreformen der Mitgliederparteien ohne durchschlagenden Erfolg: Die innerparteilichen Veränderungen von CDU und SPD seit den 1990er Jahren. In Zukunft der Mitgliederpartei, Hrsg. Uwe Jun, Oskar Niedermayer, und Elmar Wiesendahl, 187-210. Opladen: Budrich.

Jun, Uwe. 2011. Die Repräsentationslücke der Volksparteien: Erklärungsansätze für den Bedeutungsverlust und Gegenmaßnahmen. In Krise und Reform politischer Repräsentation, Hrsg. Markus Linden, Winfried Thaa, 95-124. Baden-Baden: Nomos.

Katz, Richard S., und Peter Mair. 1994. How parties organize: Change and adaptation in party organizations in Western democracies. London/Thousand Oaks: Sage.

Key, Valdemir Orlando. 1953. Politics, parties and pressure groups, 3. Aufl., New York: Thomas Y. Crowell.

Kirchheimer, Otto. 1965. Der Wandel des westdeutschen Parteisystems. Politische Vierteljahresschrift 6(1):20-41.

Kornelius, Bernhard, und Dieter Roth. 2004. Politische Partizipation in Deutschland. Ergebnisse einer repräsentativen Umfrage. Bonn: Bundeszentrale für politische Bildung.

Korte, Karl-Rudolf, Dennis Michels, Jan Schoofs, Niko Switek, und Kristina Weissenbach. 2018. Parteiendemokratie in Bewegung: Organisations- und Entscheidungsmuster der deutschen Parteien im Vergleich. Baden-Baden: Nomos.

Lamprianou, Iasonas. 2013. Contemporary political participation research: a critical assessment. In $D e$ mocracy in transition. Political participation in the European Union, Hrsg. Kyriakos N. Demetriou, 21-42. Heidelberg, New York: Springer.

Laux, Annika. 2011. Was motiviert Parteimitglieder zum Beitritt? In Parteimitglieder in Deutschland, Hrsg. Tim Spier, Markus Klein, Ulrich von Alemann, Hanna Hoffmann, Annika Laux, Alexandra Nonnenmacher, und Katharina Rohrbach, 61-78. Wiesbaden: Springer.

Lipset, Seymour Martin, und Stein Rokkan. 1967. Cleavage structures, party systems and voter alignments. An introduction. In Party systems and voter alignments. Cross-national perspectives, Hrsg. Seymour Martin Lipset, Stein Rokkan, 1-64. New York: in.

Mair, Peter, Wolfgang C. Müller, und Fritz Plasser. 2004. Political parties and electoral change: party responses to electoral markets. London: Sage.

Mays, Anja, und Verena Hambauer. 2016. Die Bedeutung von Veränderungen in der sozioökonomischen Ressourcenausstattung für das individuelle politische Engagement. Politische Psychologie 2016(2):215-235.

Montero, José R., Anders Westholm, und Jan W. van Deth. 2007. Conclusion. The realization of democratic citizenship in Europe. In Citizenship and involvement in European democracies. A comparative analysis, Hrsg. Jan W. van Deth, José R. Montero, und Anders Westholm, 415-438. London/New York: Routledge.

Morales, Laura. 2005. Attitudes, resources, opportunities, and mobilisation: a multilevel model of political membership. Working Paper 40/2005, University of Madrid: Working Paper Series, http://www.uam. es/centros/derecho/cpolitica/papers.htm.

Morales, Laura. 2009. Joining political organisations. Institutions, mobilisation and participation in western democracies. Colchester: ECPR Press.

Müller, Wolfgang C. 2000. Political parties in parliamentary democracies: making delegation and accountability work. European Journal of Political Research 37(3):309-333.

Niedermayer, Oskar. 2005. Bürger und Politik. Politische Orientierungen und Verhaltensweisen der Deutschen, 2. Aufl., Wiesbaden: Springer.

Niedermayer, Oskar (Hrsg.). 2013. Handbuch Parteienforschung. Wiesbaden: Springer.

Niedermayer, Oskar. 2016. Parteimitgliedschaften im Jahre 2015. Zeitschrift für Parlamentsfragen 47(2):411-436.

Niedermayer, Oskar. 2017. Wahlverhalten und Parteiensystem im Vorfeld der Bundestagswahl. Zeitschrift für Staats- und Europawissenschaften 15(2/3):464-484.

Panebianco, Angelo. 1988. Political parties: organization and power. Cambridge: Cambridge University Press. 
Persson, Mikael. 2015. Education and political participation. British Journal of Political Science 45(3):689-703.2.

Poguntke, Thomas. 2017. Changing or getting changed: the example of the German Greens since 1979. In Parties, governments and elites: the comparative study of democracy, Hrsg. Philipp Harfst, Ina Kubbe, und Thomas Poguntke, 87-103. Wiesbaden: Springer.

Poletti, Monica, Paul Webb, und Tim Bale. 2019. Why do only some people who support parties actually join them? Evidence from Britain. West European Politics 42(1):156-172. https://doi.org/10.1080/ 01402382.2018.1479921.

Poletti, Monica, Tim Bale, und Paul Webb. 2016. Explaining the pro-Corbyn surge in Labour's membership. blogs.lse.ac.uk/politicsandpolicy/explaining-the-pro-corbyn-surge-in-labours-membership/. Zugegriffen: 9. Apr. 2017.

Rattinger, Hans. 2009. Einführung in die politische Soziologie. München: DeGruyter Oldenbourg.

Rohrbach, Katharina. 2013. Partizipation in politischen Parteien. Eine empirische Analyse des Parteibeitritts, der Aktivität und des Parteiaustritts. Frankfurt am Main: Peter Lang.

Sartori, Giovanni. 2005. Parties and party systems: a framework for analysis. Colchester: ECPR Press.

Scarrow, Susan E. 2014. Beyond party members. Changing approaches to partisan mobilization. Oxford: Oxford University Press.

Schimank, Uwe. 2012. Sozialer Wandel. Wohin geht die Entwicklung? In Deutsche Verhältnisse. Eine Sozialkunde, Hrsg. Stefan Hradil, 17-41. Bonn: Bundeszentrale für politische Bildung.

Schlozman, Kay Lehman, Nancy Burns, und Sidney Verba. 1999. 'What happened at work today?': a multistage model of gender, employment, and political participation. The Journal of Politics 61(1):29-53.

Schlozman, Kay Lehman, Sidney Verba, und Henry E. Brady. 2013. The unheavenly chorus. Unequal political voice and the broken promise of American democracy. Princeton/Oxford: Princeton University Press.

Schmitt-Beck, Rüdiger, und Stefan Weick. 2001. Die dauerhafte Parteiidentifikation - nur noch ein Mythos? Eine Längsschnittanalyse zur Identifikation mit politischen Parteien in West- und Ostdeutschland. Informationsdienst Soziale Indikatoren 26:1-5.

Schoen, Harald, und Cornelia Weins. 2014. Der sozialpsychologische Ansatz zur Erkärung von Wahlverhalten. In Handbuch Wahlforschung, Hrsg. Jürgen W. Falter und Harald Schoen, 241-329. Wiesbaden: Springer. https://doi.org/10.1007/978-3-658-05164-8_7.

Spier, Tim. 2012. Wie aktiv sind die Mitglieder der Parteien? In Parteimitglieder in Deutschland, Hrsg. Tim Spier, Markus Klein, Ulrich von Alemann, Hanna Hoffmann, Annika Laux, Alexandra Nonnenmacher, und Katharina Rohrbach, 97-119. Wiesbaden: VS/Springer.

Spier, T., U. Alemann, H. Hoffmann, M. Klein, A. Laux, A. Nonnenmacher, und K. Rohrbach 2012. Parteimitglieder in Deutschland. Wiesbaden: VS/Springer.

Terwey, Michael, und Stefan Baltzer. 2011. ALLBUS 2008-variable report. GESIS-Variable Reports 2011/04. Köln: GESIS-Leibniz-Institut für Sozialwissenschaften.

Verba, Sidney, und Norman Nie. 1972. Participation in America: political democracy and social equality. New York: University of Chicago Press.

Verba, Sidney, Kay Lehman Schlozman, und Henry E. Brady. 1995. Voice and equality. Civic voluntarism in American politics. Cambridge: Harvard University Press.

Vetter, Angelika. 2000. Frischer Wind in einer alten Beziehung? Political efficacy und die Bundestagswahl 1998. In Wirklich ein Volk? Die politischen Orientierungen von Ost- und Westdeutschen im Vergleich, Hrsg. Jürgen W. Falter, Oscar W. Gabriel, und Hans Rattinger, 79-110. Opladen: VS/Springer.

Ware, Alan. 1992. Activist-leader relations and the structures of political parties: 'exchange' models and vote-seeking behaviour in parties. British Journal of Political Science 22(1):71-92.

Whiteley, Paul. 2011. Is the party over? The decline of party activism and membership across the democratic world. Party Politics 17(1):21-44.

Whiteley, Paul. 2012. Political participation in Britain. The decline and revival of civic culture. Basingstoke: Palgrave Macmillan.

Wiesendahl, Elmar. 2010. Zwei Dekaden Party Change-Forschung. Eine kritische Bilanz. In Krise oder Wandel der Parteiendemokratie?, Hrsg. David H. Gehne, Tim Spier, 92-120. Wiesbaden: VS/ Springer.

Wiesendahl, Elmar. 2011. Partizipation und Engagementbereitschaft in Parteien. Demokratie in Deutschland 2011 - Ein Report der Friedrich-Ebert-Stiftung, FES, Berlin. http://www.fes.de/BerlinerAkade miegespraeche/documents/ElmarWiesendahl_PartizipationundEngagementbereitschaftinParteien.pdf. Zugegriffen: 9. Apr. 2017. 